\title{
A balanced budget view on forming giant planets by pebble accretion
}

\author{
Jonathan W. Lin, ${ }^{1,2 \star}$ Eve J. Lee ${ }^{1,3 \star}$ and Eugene Chiang ${ }^{1,4 \star}$ \\ ${ }^{1}$ Department of Astronomy, University of California Berkeley, Berkeley, CA 94720-3411, USA \\ ${ }^{2}$ Department of Engineering Science, University of California Berkeley, Berkeley, CA 94720-1702, USA \\ ${ }^{3}$ TAPIR, Walter Burke Institute for Theoretical Physics, Mailcode 350-17, Caltech, Pasadena, CA 91125, USA \\ ${ }^{4}$ Department of Earth and Planetary Science, University of California Berkeley, Berkeley, CA 94720-4767, USA
}

Accepted 2018 August 5. Received 2018 July 31; in original form 2018 May 22

\begin{abstract}
Pebble accretion refers to the assembly of rocky planet cores from particles whose velocity dispersions are damped by drag from circumstellar disc gas. Accretion cross-sections can approach maximal Hill-sphere scales for particles whose Stokes numbers approach unity. While fast, pebble accretion is also lossy. Gas drag brings pebbles to protocores but also sweeps them past; those particles with the largest accretion cross-sections also have the fastest radial drift speeds and are the most easily drained out of discs. We present a global model of planet formation by pebble accretion that keeps track of the disc's mass budget. Cores, each initialized with a lunar mass, grow from discs whose finite stores of $\mathrm{mm}-\mathrm{cm}$-sized pebbles drift inward across all radii in viscously accreting gas. For every $1 M_{\oplus}$ netted by a core, at least $10 M_{\oplus}$ and possibly much more are lost to radial drift. Core growth rates are typically exponentially sensitive to particle Stokes number, turbulent Mach number, and solid surface density. This exponential sensitivity, when combined with disc migration, tends to generate binary outcomes from 0.1 to 30 au: either sub-Earth cores remain sub-Earth, or explode into Jupiters, with the latter migrating inward to varying degrees. When Jupiter-breeding cores assemble from mm-cm-sized pebbles, they do so in discs where such particles drain out in $\sim 10^{5} \mathrm{yr}$ or less; such fast-draining discs do not fit mm-wave observations.
\end{abstract}

Key words: planets and satellites: formation - protoplanetary discs.

\section{INTRODUCTION}

Gas giant planets can only form when there is still enough gas in their natal environments, i.e. when circumstellar discs are young and gas-rich. The core accretion paradigm of gas giant formation plays out in three phases (Pollack et al. 1996). Phase 1: a rocky core coagulates from disc solids. Phase 2: a gaseous envelope forms around the core, fed from the ambient disc at a rate regulated by internal cooling and contraction of the envelope. Phase 3: the planet inflates into a gas giant as the gas accretion rate onto the core 'runs away' in response to the envelope's self-gravity.

Of these three phases, perhaps the first is the least understood. There is a need for gravitational focussing of collisions between solid particles, as time-scales for coagulating a Jupiter-breeding core (having $\gtrsim$ a few Earth masses) at the stellocentric distances where Jupiters are found (a few au) are orders of magnitude longer than the 1-10 Myr lifetimes of gas discs - if collision cross-sections are only geometric and not enhanced by gravity (Goldreich, Lithwick \& Sari 2004). How much collisions are focussed depends on how much

^E-mail: jon.880@berkeley.edu (JWL); evelee@caltech.edu (EJL); echiang@astro.berkeley.edu (EC) particle velocity dispersions are damped, which, in turn, depends on particle-size distributions. These factors are not known. The problem is tied up with the longstanding mystery of planetesimal formation (Chiang \& Youdin 2010).

'Pebble accretion' makes inroads on the problem of core assembly by exploiting the ability of seed cores to attract particles small enough to have their velocity dispersions damped by aerodynamic drag from the ambient gas disc (for a comprehensive review, see Ormel 2017). Without addressing the question of the origin of the seed core (seeds as low in mass as $\sim 10^{-3} M_{\oplus}$ have been assumed), the theory of pebble accretion points out that for particles whose aerodynamic stopping times are comparable to orbital times - 'pebbles' with order-unity Stokes numbers - the accretion crosssection can approach its maximum value set by the Hill sphere of the seed core. Assembly times of Jupiter-breeding cores have been reported to range from $\sim 10^{4} \mathrm{yr}$ (Lambrechts \& Johansen 2012) to $\sim 10^{6} \mathrm{yr}$ (Lambrechts \& Johansen 2014). Pebble accretion has been hailed as a key ingredient in understanding various architectural features of planetary systems, both in Solar and extrasolar contexts, including: the dichotomy between inner terrestrial planets and outer giants (Levison, Kretke \& Duncan 2015; Morbidelli et al. 2015); the preponderance of gas giants at a few au and of ice 
giants beyond (Bitsch et al. 2015a; Bitsch, Lambrechts \& Johansen 2015b); the orbital period distribution of warm Jupiters (Ali-Dib, Johansen \& Huang 2017); and the mass distribution of planets in the TRAPPIST-1 system (Ormel, Liu \& Schoonenberg 2017).

Although pebble accretion is touted for its speed, it is perhaps less appreciated that it can be lossy. The same aerodynamic drag that brings pebbles into contact with cores also sweeps them past, as part of the background radial drift of solids from the outer to the inner disc (e.g. Weidenschilling 1977). Particles that are most easily accreted, with Stokes numbers near unity, also drift the fastest towards the star. Factoring in this background flow - tallying how many Earth masses flow under the bridge for every Earth mass netted - is one aim of this paper. We study how pebble accretion plays out in a global (1D in radius), fully time-dependent model, one that balances the disc's finite mass budget (i.e. keeps track of the various sources and sinks of solid mass) while growing a core. We consider pebble sizes ranging from 0.01 to $1 \mathrm{~cm}$, like those probed by $\mathrm{mm}-\mathrm{cm}$ wavelength images of protoplanetary discs (e.g. ALMA Partnership et al. 2015; Andrews et al. 2016), and near the maximum size that might be achievable by particle-particle sticking (Chiang \& Youdin 2010, their section 4). Aside from specifying a few model inputs such as the disc's initial solid mass, its gas mass, and its Shakura-Sunyaev $\alpha$, we make no assumption about the regime of pebble accretion that a protocore finds itself in, letting system parameters dictate which of the myriad cases outlined by Ormel \& Klahr (2010) is appropriate at any given time - in this regard, our approach is more physically conservative than other treatments. In addition to solving the equations of pebble accretion, the model also accounts for gas accretion onto cores, and orbital migration of planets by disc torques. Our goal is to assess more realistically the prospects of pebble accretion at forming various kinds of planets - Earths, Neptunes, and, in particular, Jupiters in discs resembling those observed. We focus our attention on the growth of a single core to assess whether a given disk can create even a single giant planet. We describe our model in Section 2, present results in Section 3, and summarize and look to the future in Section 4.

\section{MODEL}

We construct a global (1D in disc radius) model of planet formation. Fig. 1 presents a pictorial overview. The model tracks the evolution of a viscous gas disc (Section 2.1); the radial transport of solid particles of fixed size (Section 2.2); the assembly of a single rocky core by pebble accretion (Section 2.3); the lowering of disc gas density near the planet's orbit due to gap opening (Section 2.4); how the core stops accreting solids (Section 2.5); how cores that have stopped accreting solids subsequently accrete nebular gas ( Sections 2.6 and 2.7); and migration of the nascent planet (Section 2.8). Readers interested in a bare-bones technical summary of the underlying equations and values of input parameters can jump to Section 2.9. Table 1 lists our symbols and their meanings.

\subsection{The gas disc}

To model the gas surface density $\Sigma_{\mathrm{g}}$ as a function of radius $a$ and time $t$, we adopt the similarity solution for an isolated, viscously spreading disc (Lynden-Bell \& Pringle 1974; Hartmann et al. 1998):

$\Sigma_{\mathrm{g}}(a, t)=\frac{C a_{1}}{3 \pi v_{1} a} \mathcal{T}^{-3 / 2} \exp \left[\frac{-a / a_{1}}{\mathcal{T}}\right]$ where $a_{1}$ is a fiducial radius, $v_{1}=v\left(a_{1}\right)$ is the kinematic viscosity at $a_{1}, \mathcal{T}=t / t_{\mathrm{s}}+1$, and

$t_{\mathrm{s}}=\frac{a_{1}^{2}}{3 v_{1}}$

is a measure of the viscous diffusion time at $a_{1}$. Equation (1) presumes that the disc viscosity scales as

$v=\alpha c_{\mathrm{s}} H \propto a^{1}$

for sound speed $c_{\mathrm{s}}$, scale height $H=c_{\mathrm{s}} / \Omega$, orbital angular frequency $\Omega$, and dimensionless viscosity parameter $\alpha$ (Shakura \& Sunyaev 1973). The scaling of (3) follows, in turn, from an assumed temperature profile

$T=T_{0}\left(\frac{a}{a_{0}}\right)^{-1 / 2}$

for $a_{0}=1 \mathrm{au}$ and $T_{0}=260 \mathrm{~K}$. The normalization constant $C$ depends on an assumed initial gas mass of the disc $M_{\text {disc, }}(0)$. Given (4), the disc aspect ratio is

$H / a=0.032\left(\frac{a}{a_{0}}\right)^{1 / 4}$.

In sum, there are three free parameters: $M_{\text {disc, }}(0), \alpha$, and $a_{1}$ (equivalently, $t_{\mathrm{s}}$ ). The ranges of these and other variables are given in Table 1 .

\subsection{The solid disc}

We follow the contraction of the solid disc as its constituent particles drift inwards by gas drag. In inertial space, particles travel radially inwards at a speed

$v_{\text {drift }}=\frac{3 v}{2 a} \frac{1}{1+\tau^{2}}+2 v_{\mathrm{hw}} \frac{\tau}{1+\tau^{2}}$

where $3 v /(2 a)$ is the steady-state viscous gas velocity, $v_{\mathrm{hw}}$ is the azimuthal headwind velocity experienced by particles in pressuresupported gas, and $\tau=\Omega t_{\text {stop }}$ is the dimensionless particle stopping time (a.k.a. Stokes number). Equation (6) generalizes equation (13) of Chiang \& Youdin (2010). The headwind velocity is

$v_{\mathrm{hw}}=-\frac{c_{\mathrm{s}}^{2}}{2 \Omega a} \frac{\partial \log P_{\mathrm{g}}}{\partial \log a}$,

where the gas pressure $P_{\mathrm{g}}$ and density $\rho_{\mathrm{g}}$ are approximated as

$P_{\mathrm{g}}=\rho_{\mathrm{g}} c_{\mathrm{s}}^{2}=\frac{\Sigma_{\mathrm{g}}}{H} c_{\mathrm{s}}^{2}$.

The particle stopping time is

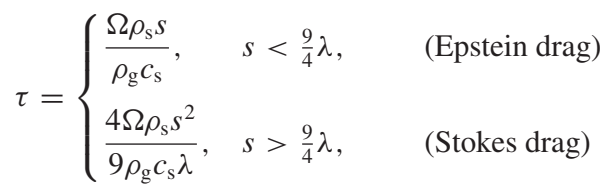

where $s$ is the particle radius, $\rho_{\mathrm{s}}=1 \mathrm{~g} \mathrm{~cm}^{-3}$ is the internal density of a single particle, and $\lambda=4 \times 10^{-9} / \rho_{\mathrm{g}}$ is the gas mean free path in cgs units. For simplicity, we take $s$ to be strictly constant in a given model (cf. Ormel \& Kobayashi 2012 who relax this assumption).

The radial transport of solids - i.e. the evolution of solid surface density $\Sigma_{\mathrm{s}}(a)$ with $t$ - is solved using a simple Lagrangian scheme. At $t=0$, the solid disc, of total mass $M_{\text {disc, } \mathrm{s}}(0)$, is divided into 1000 concentric rings that are logarithmically spaced from $a_{\text {in }}=0.01$ au to $a_{\text {out }}(0)=a_{1}$. Mass is assigned to each ring such that the initial solid surface density scales as $a^{-1}$ (following the viscously relaxed 


\section{Jupiter Formation by Pebble Accretion}

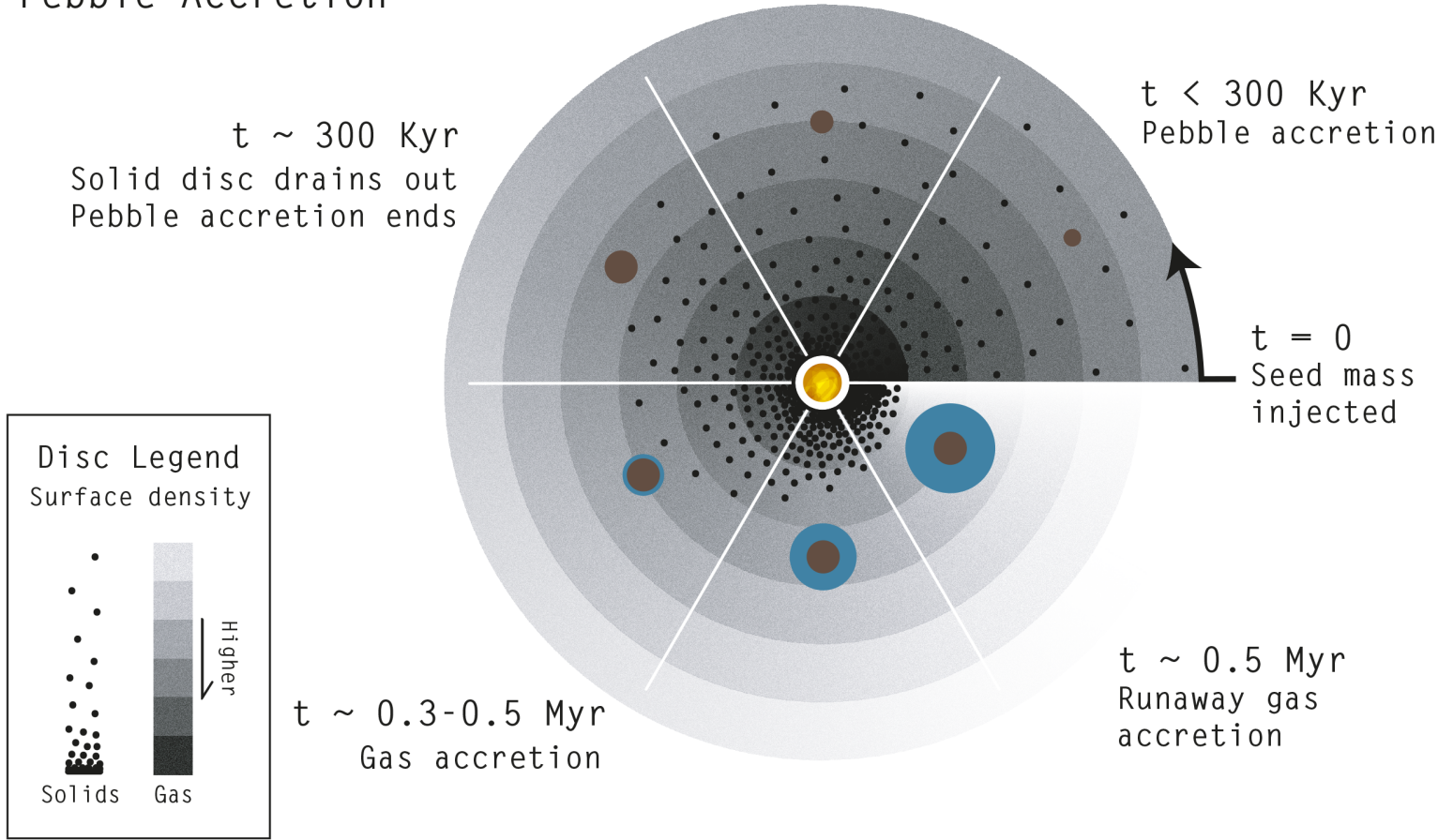

Figure 1. An example formation pathway for Jupiters, abstracted from the quantitative model shown in Fig. 8. Each sector represents a different temporal snapshot of the disc and planet, to be read counterclockwise starting from 3 o'clock. A seed core (brown) is placed in a model gas disc (grey) and begins accreting solid particles ('pebbles'; black). As pebbles feed the core, they drift inwards by aerodynamic drag exerted by the ambient gas disc. The core stops growing once the outer edge of the solid disc sweeps by. When core assembly ends and heating from pebble accretion subsides, a gas envelope (blue) grows by Kelvin-Helmholtz cooling and contraction. Eventually, the planet's gas mass becomes comparable to its core mass and the atmosphere's cooling time shortens catastrophically because of atmospheric self-gravity, triggering runaway gas accretion and inflating the planet into a gas giant. Concurrent with these processes are the inward migration of the planet and the dispersal of the gas disc (driven by viscous accretion in our model).

Table 1. Model parameters.

\begin{tabular}{|c|c|c|c|c|}
\hline Used for & Symbol & Description & Values & Reference \\
\hline \multirow[t]{4}{*}{ Gas disc } & $\alpha$ & $\begin{array}{l}\text { Viscosity parameter/turbulent Mach } \\
\text { number }\end{array}$ & $10^{-4}, 10^{-3}, 10^{-2}$ & Section 2.1 \\
\hline & $a_{1}$ & Characteristic disc radius & $10,100 \mathrm{au}$ & Section 2.1 \\
\hline & $M_{\text {disc, }, \mathrm{g}}(0)$ & Initial mass of gas disc & $10,100 M_{\mathrm{J}}$ & Section 2.1 \\
\hline & $T$ & Disc temperature & $260 \mathrm{~K} \times(a / 1 \mathrm{au})^{-1 / 2}$ & Section 2.1 \\
\hline \multirow[t]{5}{*}{ Solid disc } & $a_{\text {in }}$ & Inner radius of the solid disc & $0.01 \mathrm{au}$ & Section 2.2 \\
\hline & $a_{\text {out }}(0)$ & Initial outer radius of the solid disc & $a_{1}$ & Section 2.2 \\
\hline & $s$ & Pebble radius & $0.01,0.1,1 \mathrm{~cm}$ & Section 2.2 \\
\hline & $Z$ & Solid-to-gas mass ratio & $0.003,0.009,0.031$ & Section 2.2 \\
\hline & & $M_{\text {disc, } \mathrm{s}}(0) / M_{\text {disc, }, \mathrm{g}}(0)$ & & \\
\hline \multirow[t]{3}{*}{ Core } & $a(0)$ & Initial core orbital radius & $0.1,0.3,1,3,10,30 \mathrm{au}$ & Section 2.9 \\
\hline & $M_{\text {core }}(0)$ & Initial seed core mass & $0.01 M_{\oplus}$ & Section 2.9 \\
\hline & $M_{\text {gas }}(0)$ & Initial planet gas mass & 0 & Section 2.9 \\
\hline
\end{tabular}

Notes. (a) In selecting model parameters, we ensure that the initial core orbital radius $a(0)$ is strictly less than the characteristic disc radius $a_{1}$. In total, we have 540 unique parameter combinations. (b) Initial solid disc masses $M_{\text {disc, s }}(0)=Z M_{\text {disc, }}(0) \in\{10,30,100,300,1000\} M_{\oplus}$ (only five unique values of $M_{\text {disc, } s}(0)$ corresponding to six unique combinations of $M_{\text {disc, }}(0)$, and $Z$ ).

portion of the gas disc; Section 2.1). Each ring conserves its mass (the mass lost to pebble accretion onto the planetary core is negligible) but has its radial boundaries evolved according to equation (6). The evolution of the ring boundaries is solved using a fourth-order Runge-Kutta scheme from $t=10^{-3}$ Myr to $100 \mathrm{Myr}$ over 5000 logarithmically spaced timesteps. The solid surface density of each ring is simply the ring mass divided by the (evolving) ring area. A ring whose inner boundary crosses inside $a_{\text {in }}$ has its inner boundary held at $a_{\text {in }}$ and its surface density fixed thereafter; once the ring's outer boundary crosses $a_{\mathrm{in}}$, the ring is removed from the calculation. To reduce numerical noise when calculating $\Sigma_{\mathrm{s}}$ for a given orbital radius $a$ of the core, we use cubic splines to interpolate over the solid surface densities of up to 10 rings inside $a$, plus up to 10 rings outside (hitting disc boundaries can limit the number of rings used in the interpolation). Because the innermost ring is handled differently as per the above, it is not used in any interpolation, and 


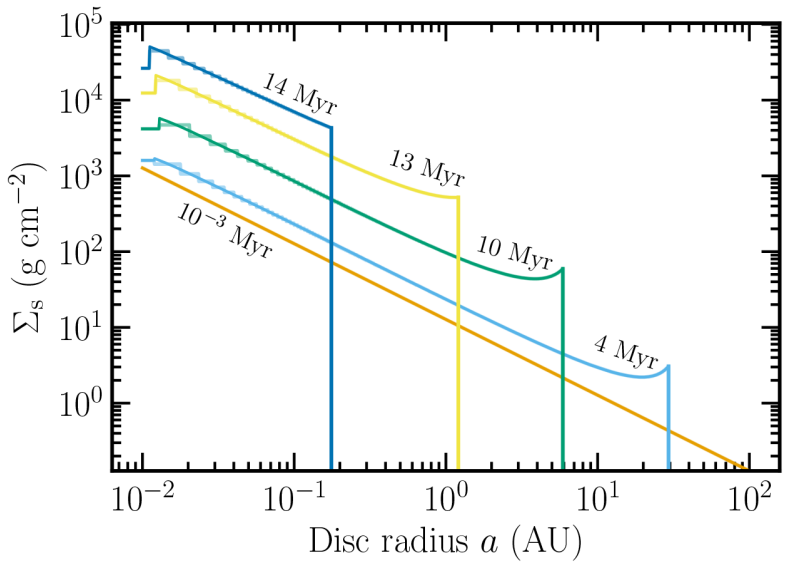

Figure 2. Surface density of solids, evolved according to our Lagrangian ring scheme (Section 2.2), for a disc with $\alpha=10^{-4}, a_{1}=100 \mathrm{au}, s=0.01 \mathrm{~cm}$, $M_{\text {disc, } \mathrm{g}}(0)=100 M_{\mathrm{J}}$, and $M_{\text {disc, } \mathrm{s}}(0)=300 M_{\oplus}$ (note that these parameters are not used in subsequent case examples but are chosen to most cleanly illustrate radial drift). Solid surface densities are interpolated over raw simulation results (step-like curves). For these model parameters, the surface density at a fixed location - say $a=1$ au - rises with time in a 'particle pile-up' before the entire solid disc sweeps by.

if the core falls within the innermost ring, its local $\Sigma_{\mathrm{s}}$ is simply that of that ring.

A sample evolution of $\Sigma_{\mathrm{s}}(a, t)$ is shown in Fig. 2. The solid disc introduces two free parameters, the particle size $s$ and the initial total solid mass $M_{\text {disc, } \mathrm{s}}(0) \equiv Z M_{\text {disc, },}(0)$ (see Table 1$)$.

\subsection{Core formation by pebble accretion}

To grow cores starting from an assumed seed mass of $M_{\text {core }}(0)=10^{-2} M_{\oplus}$, we follow the pebble accretion prescriptions of Ormel \& Klahr (2010, OK10), augmenting their formulae to account for gas turbulence (see also Ormel \& Liu 2018 and Rosenthal et al. 2018 who provide a more general treatment of stochasticity in pebble accretion). A core embedded in a disc of solids accretes mass at a rate

$\dot{M}_{\text {core }}=2 \Sigma_{\mathrm{s}} R_{\mathrm{acc}} v_{\mathrm{acc}} \times \min \left(1, R_{\mathrm{acc}} / H_{\mathrm{s}}\right)$,

where particles that come within a distance $R_{\text {acc }}$ of the core, moving at velocity $v_{\text {acc }}$ relative to the core, are accreted. According to (10), when $R_{\text {acc }}$ (the accretion 'cross section' or impact parameter) is larger than the vertical thickness of the disc of solids,

$H_{\mathrm{s}}=H \sqrt{\frac{\alpha}{\alpha+\tau}}$

(Youdin \& Lithwick 2007), then the particles effectively comprise a 2D sheet; otherwise, the thickening of the particle layer due to turbulence reduces the pebble accretion rate by a factor $R_{\mathrm{acc}} / H_{\mathrm{s}}$. For our parameter space, $\min H_{\mathrm{s}}=\min H \sqrt{\alpha / \tau} \simeq H / 40$ (characterizing only for a few models); this thickness is comparable to the physical minimum imposed by Kelvin-Helmholtz shear turbulence $\left(\sim H^{2} / a \simeq H / 30\right.$; e.g. Lee et al. 2010a,b; Rosenthal et al. 2018).

OK10 identify three regimes - settling, three-body, and hyperbolic - each having their own forms for $R_{\text {acc }}$ and $v_{\text {acc }}$. These formulae, modified for gas turbulence, are provided in the subsections below.

We erect local Cartesian axes centred on the core at orbital radius $a$, where $x$ increases radially outwards and $y$ advances in the direction of the core's (assumed circular) orbital motion. Relative to the core, the particles have mean velocity (cf. equation (6))

$v_{x}=-\frac{2 v_{\mathrm{hw}} \tau}{1+\tau^{2}}-\frac{3 v}{2 a} \frac{1}{1+\tau^{2}}-\dot{a}$,

$v_{y}=-\frac{v_{\mathrm{hw}}}{1+\tau^{2}}+\frac{3 v}{2 a} \frac{\tau}{2\left(1+\tau^{2}\right)}-\frac{3}{2} \Omega x$,

where the terms involving $\tau$ are due to drag in sub-Keplerian, viscously accreting gas (generalizations of equations (13) and (14) of Chiang \& Youdin 2010), $\dot{a}$ is the core's radial migration velocity (Section 2.8), and the term proportional to $x$ is from Keplerian shear. In addition to this mean relative velocity, gas turbulence imparts randomly oriented velocities to the particles of magnitude

$v_{\text {turb }}=c_{\mathrm{s}} \sqrt{\frac{\alpha}{1+\tau}}$

(see Youdin \& Lithwick 2007, their equation (13)). The total relative speed between the core and the particles is calculated by adding in quadrature the mean $\sqrt{v_{x}^{2}+v_{y}^{2}}$ and the fluctuation $v_{\text {turb }}$.

\subsubsection{Settling regime}

In the settling regime, particles are well-coupled to the gas $(\tau \leq 1)$. A particle is assumed to accrete onto the core if, upon approaching the core with velocity $v_{\text {acc }}$ and acquiring a specific impulse ('kick') $\Delta v$ after the encounter, its trajectory is deflected by an order-unity angle. OK10 write this condition as $\Delta v \sim v_{\text {acc }} / 4$.

For simplicity, we use an expression for $v_{\text {acc }}$ valid in the limit $\tau \ll 1$, dropping mean velocities that are typically smaller than the azimuthal headwind velocity (cf. equations (12) and (13)):

$v_{\mathrm{acc}} \sim \sqrt{\left(v_{\mathrm{hw}}+\frac{3}{2} \Omega R_{\mathrm{acc}}\right)^{2}+\alpha c_{\mathrm{s}}^{2}}$.

The settling regime is further defined by the condition that the particle stopping time be shorter than the encounter time:

$\tau / \Omega<R_{\text {acc }} / v_{\text {acc }}$.

Under this assumption, the kick velocity is

$\Delta v \sim \frac{G M_{\text {core }}}{R_{\text {acc }}^{2}} \frac{\tau}{\Omega}$,

i.e. the particle is assumed to attain terminal velocity during the encounter (with the gravitational force from the core balancing gas drag). Setting $\Delta v$ equal to $v_{\text {acc }} / 4$, and re-writing in Hill's units, we have

$\frac{9}{4} b^{6}+3 \zeta b^{5}+\left(\zeta^{2}+\alpha f_{P}^{-1} \mu^{-1 / 3} \zeta\right) b^{4}-144 \tau^{2}=0$

which we solve for $b=R_{\text {acc }} / R_{\text {Hill }}$. Here, $R_{\text {Hill }}=\mu^{1 / 3} a$, $\mu=M_{\text {core }} /\left(3 M_{\star}\right), M_{\star}$ is the stellar mass, $\zeta=v_{\text {hw }} / v_{\text {Hill }}, v_{\text {Hill }}=\Omega R_{\text {Hill }}$, and $f_{P}=-(1 / 2) \partial \log P_{\mathrm{g}} / \partial \log a$. Having solved (17) for $R_{\mathrm{acc}}$, we insert into (14) to evaluate $v_{\text {acc }}$. Our equation (17) is analogous to equation (27) of OK10 except that our accounting for turbulence in $v_{\text {acc }}$ has resulted in a higher-order polynomial.

\subsubsection{Three-body regime}

In the three-body regime, particles execute trajectories in and around the Hill sphere that are perturbed only slightly by gas drag $(\tau \gg 1)$. Accretion in this regime should be similar to that of a sub-Hill disc and its chaotic Hill sphere dynamics (Goldreich et al. 2004, their 
sections 3.3 and 3.4 ; our $2 \mathrm{D} / 3 \mathrm{D}$ correction factor $\min \left(1, R_{\mathrm{acc}} / H_{\mathrm{s}}\right)$ in equation 10 distinguishes between their 'not very thin' and 'very thin' discs). Following OK10, we take

$v_{\text {acc }}=3.2 v_{\text {Hill }}$

and

$b=1.7 \alpha_{\text {core }}^{1 / 2}+\frac{1}{\tau}$,

where $\alpha_{\text {core }} \equiv R_{\text {core }} / R_{\text {Hill }}$ (not to be confused with the gas turbulent Mach number $\alpha$ ), and the term $1 / \tau$ is OK10's empirical correction for how gas drag enhances the accretion cross section (and which can be justified using an energy argument; Rosenthal et al. 2018).

In the quasi-Hill sphere dynamics assumed by the three-body regime, the dominant velocity with which a particle approaches the core is set by the Kepler shear term (3/2) $\Omega x \sim \Omega R_{\text {Hill }}=v_{\text {Hill }}$ in equations (12) and (13). We write this defining condition as follows, assuming for simplicity that $\tau \gg 1$, and dropping the terms proportional to the viscous gas velocity $3 v /(2 a)$ and the migration velocity $\dot{a}$ which are typically negligible compared to $v_{\text {Hill }}$ :

$\sqrt{4 v_{\mathrm{hw}}^{2} / \tau^{2}+\alpha c_{\mathrm{s}}^{2} / \tau}<v_{\text {Hill }}$

which re-written in Hill's units reads

$\tau^{2}-\alpha f_{P}^{-1} \mu^{-1 / 3} \zeta \tau-\zeta^{2}>0$.

When $\alpha=0$, this reduces to the same condition $\tau \gtrsim \zeta$ used by OK10 to demarcate the three-body regime (see their fig. 7).

\subsubsection{Hyperbolic regime}

In the hyperbolic regime, particle-core encounters are fast - faster than the particle stopping time:

$R_{\text {acc }} / v_{\text {acc }}<\tau / \Omega$

in violation of the settling condition (15), and also faster than the time to cross the Hill sphere at the Hill velocity:

$R_{\text {acc }} / v_{\text {acc }}<R_{\text {Hill }} / v_{\text {Hill }}=1 / \Omega$

in violation of the Hill sphere dynamics assumed by the threebody regime. The encounter therefore unfolds in a classic two-body fashion with a gravitationally focussed accretion cross-section of

$$
\begin{aligned}
b & =\alpha_{\text {core }} \sqrt{1+\left(\frac{v_{\text {esc }}}{v_{\text {acc }}}\right)^{2}} \\
& =\alpha_{\text {core }} \sqrt{1+\frac{6}{\alpha_{\text {core }}\left(v_{\text {acc }} / v_{\text {Hill }}\right)^{2}}},
\end{aligned}
$$

where $v_{\text {esc }}$ is the escape velocity from the core surface and $v_{\text {esc }} / v_{\text {Hill }}=\sqrt{6 / \alpha_{\text {core }}}$. In general, the relative velocity $v_{\text {acc }}$ equals $\sqrt{v_{x}^{2}+v_{y}^{2}+v_{\text {turb }}^{2}}$; but in evaluating (12b) for $v_{y}$, we may drop the Kepler shear term since $b \ll 1$ and $\zeta=v_{\text {hw }} / v_{\text {Hill }}>1$ in the hyperbolic regime. Thus

$$
\begin{aligned}
\frac{v_{\mathrm{acc}}}{v_{\mathrm{Hill}}} \sim & {\left[\left(\frac{2 \zeta \tau}{1+\tau^{2}}+\frac{3}{2} \frac{\alpha \zeta f_{P}^{-1}}{1+\tau^{2}}+\frac{\dot{a}}{v_{\text {Hill }}}\right)^{2}\right.} \\
& \left.+\left(\frac{\zeta}{\left(1+\tau^{2}\right)}-\frac{3}{2} \frac{\alpha \zeta f_{P}^{-1} \tau}{2\left(1+\tau^{2}\right)}\right)^{2}+\frac{\alpha \zeta \mu^{-1 / 3}}{f_{P}(1+\tau)}\right]^{1 / 2}
\end{aligned}
$$

This matches equation (29) of OK10 when $\alpha=0$ and $\dot{a}=0$.
We summarize in Fig. 3 our decision tree for choosing the appropriate regime of pebble accretion as we track the growth of a core. When the hyperbolic regime is selected, we verify a posteriori that the inequalities (22) and (23) are satisfied. In practice, we find for our model parameters that pebble accretion is almost always in the settling regime (cf. Fig. 15 which uses a disjoint set of model inputs and which shows some cases of hyperbolic accretion). As noted in Section 2.2, in evaluating $\Sigma_{\mathrm{s}}$ in the accretion rate (10), we interpolate using cubic splines in the vicinity of the core.

\subsection{Opening a gap in the gas disc}

A core exerts Lindblad torques on the gas disc that open an annular gap about its orbit. The gas surface density at the position of the planet, $\Sigma_{\mathrm{g} \text {, planet }}$, is related to the local unperturbed value $\Sigma_{\mathrm{g}}$ by

$$
\frac{\Sigma_{\mathrm{g}}}{\Sigma_{\mathrm{g}, \text { planet }}}-1=0.043\left(\frac{M_{\text {planet }}}{M_{\star}}\right)^{2}\left(\frac{H}{a}\right)^{-5} \alpha^{-1}
$$

(Dong \& Fung 2017; see also Kanagawa et al. 2015 and Fung, Shi \& Chiang 2014). Here, $M_{\text {planet }}=M_{\text {core }}+M_{\text {gas }}$ is the total planet mass, including not only the rocky core but also any gas envelope it carries (accretion of gas onto the core is treated in Section 2.6), while $M_{\star}=\mathrm{M}_{\odot}$ is the host star mass.

Depressing the gas surface density at the position of the planet has two effects. First, it reduces - very slightly - the rate at which the solid core accretes a gas envelope (see the weak dependence on $\Sigma_{\mathrm{g} \text {, planet }}$ in equation (28)). Second and more significantly, once the gas gap becomes deep enough, orbital migration of the planet is expected to slow from Type I to Type II (Section 2.8).

\subsection{Halting pebble accretion}

As a planet grows in mass and opens a deeper gap in the gas disc, local pressure gradients near gap edges may trap particles there. Lambrechts \& Johansen (2014, LJ14) term the core mass above which solids can no longer drift onto the core the "pebble isolation mass.' In hydrodynamics simulations, the pebble isolation mass is found to scale as the thermal mass (that for which surface density perturbations excited by the planet become non-linear):

$M_{\text {peb,iso }}=5 M_{\oplus}\left(\frac{H / a}{0.03}\right)^{3}\left[0.34\left(\frac{-3}{\log _{10} \alpha}\right)^{4}+0.66\right]$.

(Bitsch et al. 2018; see also equations (33) and (36) of Dipierro \& Laibe 2017; Ataiee et al. 2018; Rosotti et al. 2016;LJ14).

In our model, a core stops accreting particles either when (a) $M_{\text {planet }}>M_{\text {peb, iso }}$, or (b) the entire disc of solids has drifted past the core (Section 2.2). Outcome (b) will prove common.

\subsection{Gas accretion onto cores}

Cores accrete as much gas as can cool - which it cannot while pebble accretion is ongoing, as the rate of accretional heating tends to exceed the rate of envelope cooling by orders of magnitude (Fig. 4). We therefore start gas accretion onto solid cores only after the cores have finished accreting pebbles (Section 2.5).

We adopt the scaling relation for atmospheric growth derived by Lee \& Chiang (2015, their equation (24), derived for temperatures between $100 \mathrm{~K}$ and $800 \mathrm{~K}$ ), accounting for the weak dependence on nebular surface density (Lee \& Chiang 2016, their fig. 4; we have 


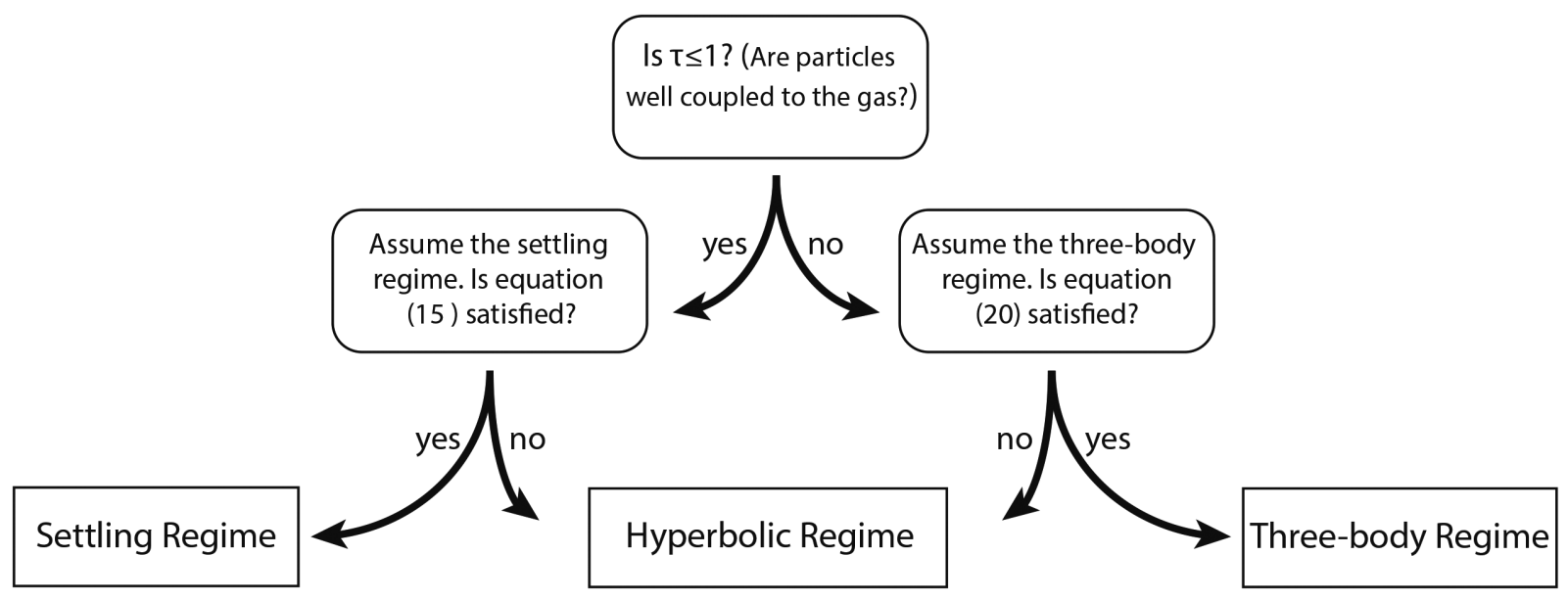

Figure 3. Flowchart for choosing the appropriate pebble accretion regime. In the case where the hyperbolic regime is returned, we perform additional $a$ posteriori checks given by equations (22) and (23). In practice, for our parameters, pebble accretion is nearly always in the settling regime.

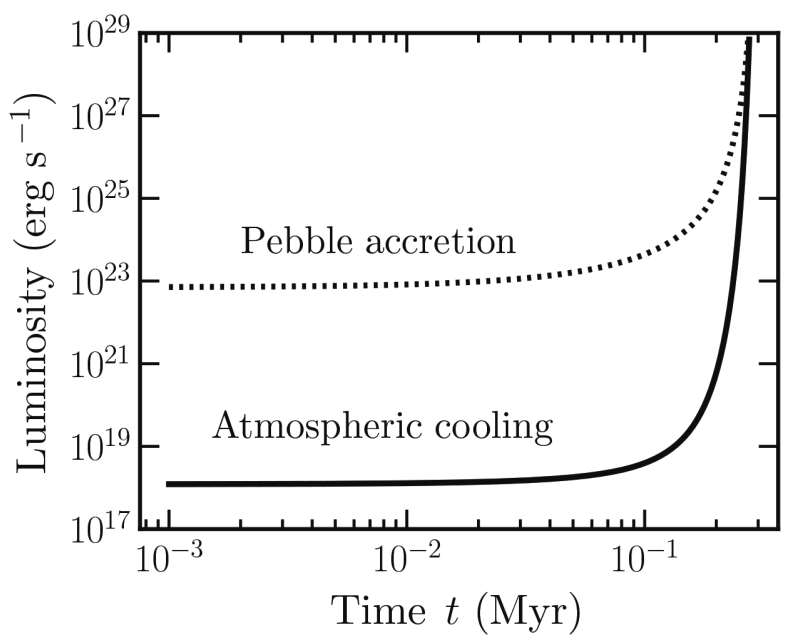

Figure 4. Pebble accretion luminosities tend to overwhelm atmospheric cooling luminosities, preventing gas from accreting onto solid cores until after pebbles have stopped accreting. The pebble accretion luminosity (dotted curve) is computed as $G M_{\text {core }} \dot{M}_{\text {core }} / R_{\text {core }}$, where $\dot{M}_{\text {core }}$ is computed using (10) and $R_{\text {core }}$ derives from $M_{\text {core }}$ assuming a fixed bulk density of $5 \mathrm{~g} \mathrm{~cm}^{-3}$. The core mass is initially set to $0.01 M_{\oplus}$ at a time of $10^{-3} \mathrm{Myr}$, and accretes pebbles with size $s=0.1 \mathrm{~cm}$ at a nearly constant $a=1 \mathrm{au}$, in a disc for which $\alpha=10^{-4}, a_{1}=100 \mathrm{au}$, and the initial gas and solid masses are $M_{\text {disc, }}(0)=10 M_{\mathrm{J}}$ and $M_{\text {disc, } \mathrm{s}}(0)=100 M_{\oplus}$ (these same disc parameters are adopted for Fig. 8). The core grows until the solid disc drifts entirely inside of the core's orbit, at which point $M_{\text {core }} \simeq 3 M_{\oplus}$. To compute the atmospheric cooling luminosity (solid curve), we use Lee \& Chiang (2015, their equations (8) and afterward), with $T_{\text {rcb }}$ set equal to $260 \mathrm{~K}$, opacity constants appropriate to their section 2.2.2 for dust-free and gas-rich discs, and the gas-to-core mass ratio fixed at GCR $=0.01$; assuming a higher GCR only lowers the cooling luminosity and strengthens the point of this plot.

verified that this density dependence applies at different distances):

$$
\begin{aligned}
\frac{M_{\text {gas }}}{M_{\text {core }}}= & 0.20\left(\frac{\Delta t}{0.1 \mathrm{Myr}}\right)^{0.4}\left(\frac{500 \mathrm{~K}}{T}\right)^{1.5} \\
& \times\left(\frac{M_{\text {core }}}{5 M_{\oplus}}\right)^{1}\left(\frac{\Sigma_{\text {g,planet }}}{0.03 \Sigma_{\text {mmen }}}\right)^{0.12},
\end{aligned}
$$

where $M_{\text {gas }}$ is the planetary gas mass, $\Delta t$ is the elapsed time since the onset of gas accretion, $T$ is the nebular temperature, and $\Sigma_{\text {mmen }}$ is the surface density of the minimum-mass extrasolar nebula (Chiang \& Laughlin 2013):

$$
\Sigma_{\text {mmen }}=4 \times 10^{5}\left(\frac{a}{0.1 \mathrm{au}}\right)^{-1.6} \mathrm{~g} \mathrm{~cm}^{-2} .
$$

Equation (28) drops the contribution from any dust to the gas opacity; this is a plausible assumption given grain coagulation, settling, and ablation (Ormel 2014; Brouwers, Vazan \& Ormel 2018). ${ }^{1} \mathrm{We}$ also ignore for simplicity the effects of super-solar gas-phase metallicity, which can hasten the rate of cooling and gas accretion, but only for metallicities $Z \gtrsim 0.5$ (e.g. Venturini et al. 2015, their fig. 1; see also Venturini \& Helled 2017).

Equation (28) further presumes that the bulk of the envelope mass is centrally concentrated near the core, i.e. the adiabatic index $\gamma_{\text {ad }}<4 / 3$ in the inner convective zone. This assumption is valid when envelope temperatures $>2500 \mathrm{~K}$ so that $\mathrm{H}_{2}$ dissociates. Only cores more massive than about $0.5 M_{\oplus}$ can gravitationally retain such gas, and so we apply equation (28) to grow atmospheres only for cores which exceed $0.5 M_{\oplus}$ at the end of the pebble accretion phase. ${ }^{2}$ For such cores, we combine the time derivatives of equation (28) with the planet's time-varying orbital distance (Section 2.8) and the disc's time-varying surface density (Section 2.1) to track increments in the planet's gas mass as ambient nebular conditions change. In taking the time derivative of (28), we ignore terms depending on $d T / d t$ and $d \Sigma_{\mathrm{g} \text {, planet }} / d t$ for simplicity. We also check that the envelope mass does not exceed the value that it would have if it were isothermal at the disc temperature; this is the maximum mass to which the atmosphere can grow, as gas cannot cool past this point (see Lee \& Chiang 2015, their fig. 4). If the envelope mass exceeds this isothermal bound, we halt gas accretion.

\footnotetext{
${ }^{1}$ Lambrechts \& Lega (2017) argue otherwise, that dust from disc gas is continuously brought to the planet's outer envelope. We therefore also experiment by replacing equation (28) with an analogous formula based on an opacity that includes dust (Lee \& Chiang 2015, their equation (20)). None of the conclusions of this paper changes quantitatively; more details on this separate set of experiments are given in the Results section, footnote 3 .

${ }^{2}$ We have also determined, following the methodology of Lee, Chiang \& Ormel (2014), that core masses $<0.5 M_{\oplus}$ take longer than $\sim 10$ Myrs to accrete even 1 per cent-by-mass envelopes.
} 


\subsection{Runaway gas accretion and the formation of Jupiters}

Once the gas envelope mass becomes comparable to the underlying core mass, the envelope's self-gravity becomes significant. Selfgravitating envelopes demand larger luminosities to sustain hydrostatic equilibrium; as gas continues to pile on, the cooling time-scale shortens catastrophically, and the envelope grows in a runaway fashion (e.g. Pollack et al. 1996). We crudely model runaway using a step function: once $M_{\text {gas }} / M_{\text {core }} \geq 0.5$, we boost $M_{\text {planet }}=M_{\text {gas }}+$ $M_{\text {core }}$ to $1 M_{\mathrm{J}}$, or we add to $M_{\text {gas }}$ the total mass in the gas disc outside the planet's orbit, whichever option yields a smaller $M_{\text {planet }}$. The latter option is not to be taken literally (i.e. we do not literally set $\Sigma_{\mathrm{g}}$ to zero outside the planet's orbit), but is merely a rough proxy for mass conservation.

\subsection{Disc-driven migration}

We define a 'deep' gap as one for which $\Sigma_{\mathrm{g} \text {, planet }}=1 / 10$ the value of the unperturbed surface density $\Sigma_{\mathrm{g}}$. From (26), deep gaps are opened by planet masses exceeding

$$
\begin{aligned}
M_{\text {Type II }} & =14.5 M_{\star} \alpha^{1 / 2}\left(\frac{H}{a}\right)^{5 / 2} \\
& =8 M_{\oplus}\left(\frac{\alpha}{10^{-4}}\right)^{1 / 2}\left(\frac{H / a}{0.03}\right)^{5 / 2} .
\end{aligned}
$$

Planets for which $M_{\text {planet }}<M_{\text {Type II }}$ are transported radially according to the Type I migration rate:

$\dot{a}=\frac{2 \Gamma_{\text {tot }}}{M_{\text {planet }} \Omega a}$,

where $\Gamma_{\text {tot }}$ is the combined Lindblad and corotation torque exerted by the disc on the planet (e.g. Kley \& Nelson 2012):

$\Gamma_{\text {tot }} \approx-2.2\left(\frac{M_{\text {planet }}}{M_{\star}}\right)^{2}\left(\frac{a}{H}\right)^{2} \Sigma_{\text {g,planet }} a^{4} \Omega^{2}$.

All quantities are evaluated at the location of the planet. The numerical pre-factor is calibrated using the hydrodynamical simulations of D'Angelo \& Lubow (2010) for the power-law indices describing our gas surface density and temperature profiles. For $M_{\text {planet }}>M_{\text {Type II }}$, we switch the migration rate from Type I to Type II:

$\dot{a}=-\frac{\alpha c_{\mathrm{s}} H}{a} \times \min \left(1, \Sigma_{\mathrm{g}} a^{2} / M_{\text {planet }}\right)$.

The correction factor $\min \left(1, \Sigma_{\mathrm{g}} a^{2} / M_{\text {planet }}\right)$ accounts for how the planet adds an extra load to the disc whose viscous drift rate is then slowed (note that we assume $\Sigma_{\mathrm{g}}$ and not $\Sigma_{\mathrm{g} \text {, planet }}$ is relevant for this correction). Duffell et al. (2014) point out that the actual Type II rate could differ from (33) by factors of several because disc gas can cross the gap (see also Kanagawa, Tanaka \& Szuszkiewicz 2018 for an improved treatment of how gap-opening planets migrate); we neglect this effect.

\subsection{Implementation summary}

There are five input parameters governing the disc: $\alpha, a_{1}, s$, $M_{\text {disc, } \mathrm{g}}(0)$, and $Z \equiv M_{\text {disc, } \mathrm{s}}(0) / M_{\text {disc, } \mathrm{g}}(0)$. For the planet, we have another three inputs: the initial core mass $M_{\text {core }}(0)$, the initial gas mass $M_{\text {gas }}(0)$, and the initial orbital radius $a(0)$. For all simulations, we fix $M_{\text {core }}(0)=10^{-2} M_{\oplus}$ and $M_{\text {gas }}(0)=0$. All other parameters are systematically varied across 540 different models; see Table 1.

Equation (10), the time derivative of equation (28), and equation (31) (or equation (33) once $M_{\text {planet }}>M_{\text {Type II }}$ ) define a coupled

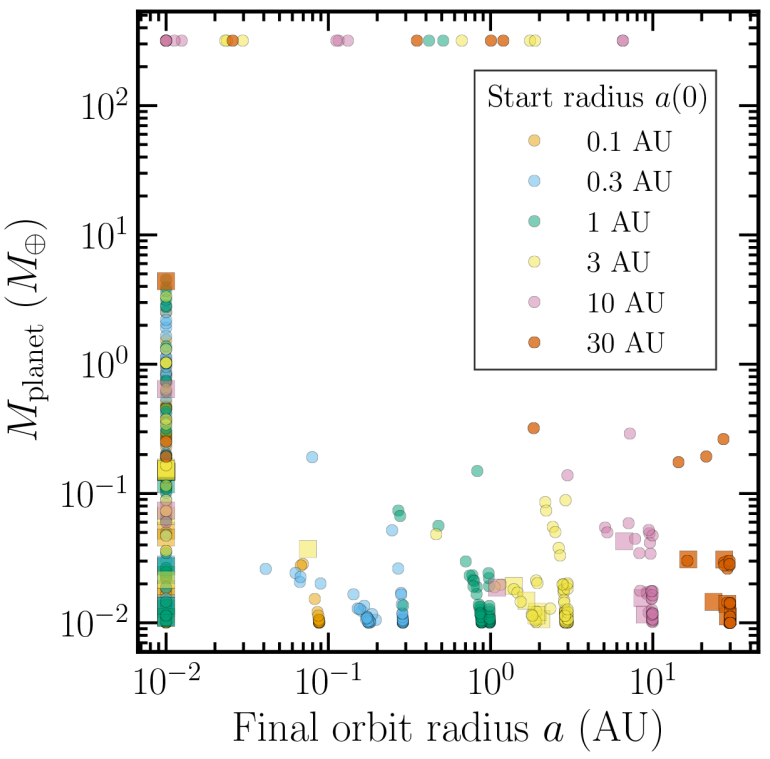

Figure 5. Final planet mass versus final orbital distance for each of 540 parameter configurations (see Table 1). Each point is coloured according to the initial position of the seed core $a(0)$. Those models with a solid drift time-scale, $a_{1} /\left[v_{\mathrm{drift}}\left(a_{1}, 0\right)\right]$, longer than $1 \mathrm{Myr}$ are marked as squares, while discs with shorter drift time-scales are marked as circles. Certain parameter choices lead to cold, warm, and hot Jupiters (top line of points), all of which experience migration, and none of which materialize from long-lived solid discs (no squares, only circles). No super-Earths/sub-Neptunes form outside of the innermost disc edge at $0.01 \mathrm{au}$; we are inclined to rule out those models where super-Earths are found at the innermost edge at $a=0.01$ au having migrated there, as such models would predict short-period pile-ups in superEarth occurrence rates that are not observed (Lee \& Chiang 2017). Over most of our parameter space, cores hardly grow, remaining $\lesssim 0.1 M_{\oplus}$.

system of differential equations for the time evolution of the planet's core mass, gas mass, and orbital radius. The background gas disc evolves according to (1), and the background solid disc is evolved according to the Lagrangian ring scheme described at the end of Section 2.2. Each model is evolved from $t=10^{-3} \mathrm{Myr}$ to $100 \mathrm{Myr}$, with state variables recorded at $5000 \mathrm{log}$-spaced times. To advance from one recording time to the next, we first evolve our background solid disc using a fourth-order Runge-Kutta method, and then solve for the planet variables using Sci Py's odeint. A core stops growing in solid mass either once it reaches the pebble isolation mass (equation (27)) or the disc of solids drifts wholly interior to the core's orbit. Once the planet's gas mass reaches half its core mass, then either $M_{\text {planet }}$ is manually set to $1 M_{\mathrm{J}}$, or $M_{\text {gas }}$ is augmented by the gas disc mass outside the planet's orbit at that time, whichever yields the smaller planet mass. Planets that migrate to our inner disc radius of $a_{\mathrm{in}}=0.01 \mathrm{au}$ are held there.

\section{RESULTS}

Fig. 5 summarizes our model outcomes. Beyond $\sim 0.1$ au - in those regions where the majority of exoplanets are detected - we find that sub-Earth cores either remain sub-Earths, or explode into gas giants. There is no in-between; super-Earths are completely absent outside the assumed disc edge at 0.01 au. Over most of the parameter space that we chart, sub-Earths are the norm: they outnumber gas giants by about 20 to 1 . Moreover, these sub-Earths have hardly grown from their initial assumed seed masses of $10^{-2} M_{\oplus}$; on the whole, they have increased their mass by factors of several at most. 
A small fraction (6 per cent) of models produce super-Earths $\left(\sim 1-10 M_{\oplus}\right)$. Formed overwhelmingly at short distances $(a(0) \leq$ $1 \mathrm{au}$ ) and/or in gas-heavy discs, the cores of these super-Earths rapidly migrate and become stranded at the disc's innermost edge, ${ }^{3}$ where the surrounding nebula is so hot that gas accretion onto cores is stunted - the atmospheric masses hit their isothermal upper bounds (evaluated at $T=2600 \mathrm{~K}$ at $a=0.01 \mathrm{au}$ ). Final gas-tocore mass ratios are about $1-10$ per cent, with a few reaching up to 30 per cent. These migration-heavy models predict that planets pile up at short orbital periods (see, e.g. Ida \& Lin 2008). Because such pile-ups are not seen in observations (see, e.g. Lee \& Chiang 2017, and references therein), we tend to discount as unrealistic those model parameters that lead to such wholesale migration of cores.

Whether a core nucleates a gas giant depends on its mass: more massive cores accrete gas faster (Pollack et al. 1996; Piso \& Youdin 2014; Lee \& Chiang 2015; Ginzburg, Schlichting \& Sari 2016). In the following, we describe analytically the factors that determine how quickly and to what final mass a core grows by pebble accretion - including how much disc mass is expended in the process. These pencil-and-paper considerations help to explain our numerical results, which are also fleshed out in greater detail below.

The final mass of the core is determined by how many pebbles the seed core nets from the background drift of solids (orbital migration of the core can be safely neglected during this early growth phase). From equation (6) for $v_{\text {drift }}$, the time it takes for the solid disc to drain from its initial outer radius $a_{1}$ to the location of the core $a$ is

$t_{\text {drift }} \simeq \begin{cases}a /\left(2 v_{\mathrm{hw}} \tau\right) \ln \left(a_{1} / a\right), & \tau \gg \alpha, \\ (2 / 3) \alpha^{-1}\left(\Omega a / c_{\mathrm{s}}^{2}\right)\left(a_{1}-a\right), & \tau \ll \alpha,\end{cases}$

where all unsubscripted variables (here and below) are evaluated at the position of the core. We have taken the limit $\tau \ll 1$ (valid over practically all of our parameter space) and made use of the fact that for our assumed disc gas surface density and temperature profiles, $\tau \propto a$ and $v_{\mathrm{hw}}=$ constant.

Because pebble accretion for our parameters occurs mostly in the settling regime ( $\tau<1$ ), and with $R_{\mathrm{acc}}<H_{\mathrm{s}}$ (see the appendix for a more general exposition; see also Ormel 2017), we can write:

$$
\begin{aligned}
\dot{M}_{\text {core }} & =2 \Sigma_{\mathrm{s}} R_{\mathrm{acc}}^{2} v_{\mathrm{acc}} / H_{\mathrm{s}} \\
& =\frac{8 \Sigma_{\mathrm{s}} G M_{\mathrm{core}} \tau}{c_{\mathrm{s}}}\left(\frac{\alpha+\tau}{\alpha}\right)^{1 / 2} .
\end{aligned}
$$

Accordingly, the core grows exponentially with time:

$M_{\text {core }}(t)=M_{\text {core }}(0) \exp \left[\frac{8 G \tau}{c_{\mathrm{s}}}\left(\frac{\alpha+\tau}{\alpha}\right)^{1 / 2} \int_{0}^{t} \Sigma_{\mathrm{s}} d t\right]$

assuming $\tau$ at the position of the core is constant with time (which it approximately is in our models at early times; see our later figures). For $\tau \gg \alpha$, we can use the relations $v_{\text {drift }} \propto v_{\text {hw }} \tau \propto a$ (Epstein) and $\Sigma_{\mathrm{s}} \propto a^{-1}$ to solve the continuity equation for $\Sigma_{\mathrm{s}}$ by separation of variables: $\Sigma_{\mathrm{s}}(a, t)=f(a) g(t) \propto a^{-1} g(t)$, where $g(t)=\exp \left[t /\left(a / v_{\text {drift }}\right)\right]$ (i.e. an exponentially rising 'particle pile-up' at fixed location; see,

\footnotetext{
${ }^{3}$ When our standard prescription for fast dust-free gas accretion is replaced with a slower one using opacities that include dust (footnote 1), those models that previously formed gas giants mostly form super-Earths that migrate and pile up at the innermost disc edge. The remainder continue to form gas giants with no substantive change in outcome (this is the case when solid disc masses and initial core distances are large).
}

e.g. Fig. 2). Then, the final core mass after $t=t_{\text {drift }}$ is

$$
\begin{aligned}
M_{\text {core }} \sim M_{\text {core }}(0) \exp \left[\frac{2}{\pi}\left(\frac{\tau}{\alpha}\right)^{1 / 2} \frac{M_{\mathrm{disc}, \mathrm{s}}(0)}{M_{\star}}\left(\frac{\Omega a}{c_{\mathrm{s}}}\right)\left(\frac{\Omega a}{v_{\mathrm{hw}}}\right)\right], \\
\tau \gg \alpha
\end{aligned}
$$

where we have used $M_{\text {disc, } \mathrm{s}}(0)=2 \pi \Sigma_{\mathrm{s}}(a, t=0) a a_{1}$. For $\tau \ll \alpha, \Sigma_{\mathrm{s}}$ is approximately constant in time (since $\Sigma_{\mathrm{s}} \propto a^{-1}$ is the steady-state solution for $v_{\text {drift }}=3 v /(2 a)=$ constant $)$, and so

$$
\begin{aligned}
M_{\text {core }} \sim & M_{\text {core }}(0) \exp \left[\frac{8}{3 \pi}\left(\frac{\tau}{\alpha}\right) \frac{M_{\text {disc, }}(0)}{M_{\star}}\left(\frac{\Omega a}{c_{\mathrm{s}}}\right)^{3} \frac{a_{1}-a}{a_{1}}\right], \\
\tau \ll \alpha &
\end{aligned}
$$

From equations (37) and (38), it follows that more massive cores, and by extension Jupiters, favour high $M_{\text {disc, } \mathrm{s}}(0)$, high $\tau$ (to enable particles to more easily 'peel off' the gas flow and fall onto the core), and low $\alpha$ (to reduce the particle scale height and increase the solid particle density; and, in cases where the pebble drift speed is determined by viscosity, to slow that drift speed down and prolong the time over which the core accretes). Fig. 6 verifies these dependencies, at least in sign. More massive cores are formed in discs with higher overall solid mass (lower left panel), smaller $\alpha$ (upper left panel), larger pebble size $s$ (which increases $\tau$; upper right panel and equation 9), and larger $a_{1}$ (which also increases $\tau$ by spreading a given gas mass over a larger area to reduce the gas density; lower right panel and equation (9)).

Equations (37) and (38) further suggest that the ratio $\tau / \alpha$ is a key parameter controlling how massive cores can grow. Fig. 7 bears this out by showing that Jupiter-breeding cores only form when $\tau / \alpha>0.1$. The condition $\tau / \alpha>0.1$ is necessary but not sufficient to create gas giants; the vast majority of our runs at $\tau / \alpha>0.1$ yield only sub-Earths, mostly because $M_{\text {disc, s }}(0)$ is too small. Another necessary condition for spawning giants is that their cores grow outside $\sim 0.1$ au - sufficiently far away from their host stars that they do not run up against the pebble isolation mass, which decreases with decreasing distance to the star $\left(M_{\text {peb, iso }} \propto a^{3 / 4}\right.$; see equations (27) and (5)). This limitation set by pebble isolation argues against in-situ formation of hot Jupiters. An example of core growth stifled by pebble isolation will be given below.

A core accretes only a fraction of the solids from the outer disc that converge onto its orbit. The remaining fraction drifts past the core into the inner disc and is 'wasted'. To grow a core from an initial seed mass $M_{\text {core }}(0)$ requires a mass investment of

$M_{\mathrm{drift}}=\int_{M_{\mathrm{core}}(0)}^{M_{\mathrm{core}}} \epsilon^{-1} d M_{\mathrm{core}}$

where the instantaneous pebble accretion efficiency

$$
\begin{aligned}
\epsilon & \equiv \frac{\dot{M}_{\text {core }}}{2 \pi \Sigma_{\mathrm{s}} v_{\text {drift }} a} \\
& =\frac{4}{\pi}\left(\frac{\Omega a}{v_{\text {drift }}}\right)\left(\frac{\Omega a}{c_{\mathrm{s}}}\right)\left(\frac{M_{\text {core }}}{M_{\star}}\right) \tau\left(\frac{\alpha+\tau}{\alpha}\right)^{1 / 2} .
\end{aligned}
$$




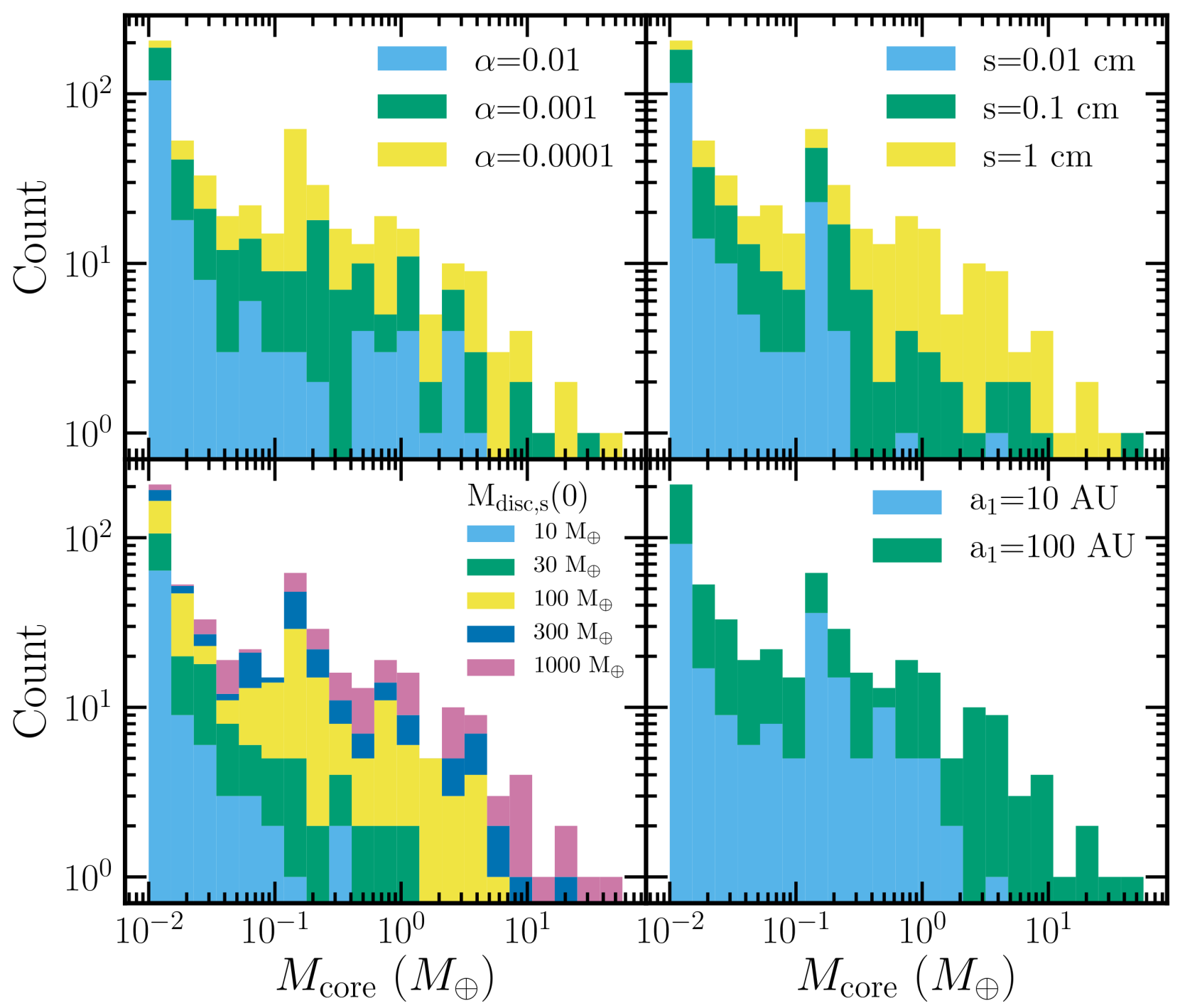

Figure 6. Final core mass histograms, stacked and coloured against various model input parameters. We see that higher mass cores prefer lower $\alpha$, higher $s$, higher $M_{\text {disc, } \mathrm{s}}(0)$, and higher $a_{1}$. See text for discussion.

Note how $\epsilon$, and by extension $M_{\text {drift }}$, do not depend on $\Sigma_{\mathrm{s}}$ and the details of its time evolution. Evaluation gives:

$$
\begin{aligned}
M_{\text {drift }}= & \frac{\pi}{4}\left(\frac{v_{\text {drift }}}{\Omega a}\right)\left(\frac{c_{\mathrm{s}}}{\Omega a}\right) \tau^{-1}\left(\frac{\alpha}{\alpha+\tau}\right)^{1 / 2} M_{\star} \ln \left[\frac{M_{\text {core }}}{M_{\text {core }}(0)}\right] \\
& \sim \begin{cases}35 M_{\oplus}\left(\frac{a}{1 \mathrm{au}}\right)^{3 / 4}\left(\frac{10}{\tau / \alpha}\right)^{1 / 2} \ln \left(\frac{M_{\text {core }} / M_{\text {core }}(0)}{100}\right), & \tau \gg \alpha \\
600 M_{\oplus}\left(\frac{a}{1 \mathrm{au}}\right)^{3 / 4}\left(\frac{0.1}{\tau / \alpha}\right) \ln \left(\frac{M_{\text {core }} / M_{\text {core }}(0)}{100}\right), & \tau \ll \alpha .\end{cases}
\end{aligned}
$$

These analytic estimates appear consistent with our numerical results as reported in Fig. 6 (lower left panel).

Example evolutionary tracks of seed cores that grow to gas giants are presented in Figs. 8 (warm Jupiter), 9 (cold Jupiter), and 10 (hot Jupiter). For comparison, we also show simulations that terminate in sub-Earths in Figs. 11, 12, and 13, illustrating three different modes by which gas giant formation by pebble accretion can fail (low $M_{\text {disc, s }}(0)$; low $M_{\text {peb, iso }}$; and $\tau / \alpha<0.1$, respectively).

For all evolutionary tracks, we verify that the core mass grows at least exponentially fast. Note how in those runs for which $\tau>\alpha$ (Figs. 8-12), the solid surface density $\Sigma_{\mathrm{s}}$ at the location of the core rises with time just before the solid disc drifts entirely past the core. This 'particle pile-up' - a traffic jam in disc solids - occurs whenever the particle drift velocity $v_{\text {drift }}$ decreases sufficiently fast with decreasing radius (Youdin \& Chiang 2004). We have verified that such an inwardly decreasing velocity profile obtains when $\tau / \alpha>0.1-$ so that the drift velocity in (6) is not dominated by viscous diffusion but has a significant contribution from aerodynamic drag - and when that drag is in the Epstein regime for $\tau \ll 1$. The latter drag regime typically holds between $\sim 0.1$ and $\sim 10$ au for our model parameters; outside $\sim 10 \mathrm{au}, \tau$ approaches unity and the velocity profile flattens, while inside $\sim 0.1$ au, Stokes drag obtains, which bleeds particles from the inside out. Where there is a particle pile-up, core growth is super-exponentially fast (equation (36)).

Although we have identified model parameters that succeed in forming Jupiters by pebble accretion, these same parameters encounter difficulty when confronted with millimetre-wavelength observations of discs. The problem is that rapid core growth by pebble accretion demands large $\tau$ (equations (37) and (38)), but that same large $\tau$ leads to solids draining quickly from the disc (equation (34)) - too quickly when compared against observations. In all of our Jupiter-forming runs, solids drain out in $\sim 0.1-0.3 \mathrm{Myr}$ (Figs 5 


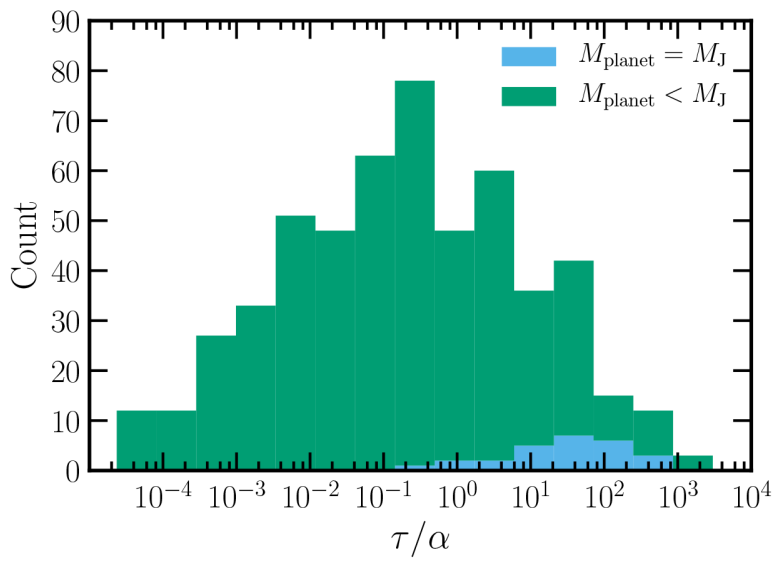

Figure 7. Histogram of the ratio $\tau / \alpha$ for our 540 models, where Stokes number $\tau$ is evaluated at the initial time (and, in practice, remains constant over most if not all of the duration of core growth; see following figures). The histogram is additionally stacked and coloured according to whether or not a gas giant emerges from a given model. All gas giants arise from model parameter combinations for which $\tau / \alpha>0.1$. This necessary condition on core growth is consistent with analytic calculations; see discussion surrounding equations (37) and (38).

and 8-10) or shorter (data not shown). These results cannot be immediately reconciled with observed discs that orbit stars 1-10-Myr old and that exhibit mm-wave continuum emission - presumably from mm-sized solids - on scales of 10-100 au (e.g. Brauer et al. 2007; Pérez et al. 2015; Tazzari et al. 2016; Tripathi et al. 2017).

\section{SUMMARY AND DISCUSSION}

We have studied how planets can form by pebble accretion, starting from an assumed seed mass of $10^{-2} M_{\oplus}$ and working our way to cores massive enough to nucleate gas giants. Our model is global in the sense that it accounts for the parent disc over its entire radial extent. We calculated how solids drift from large to small orbital radius by aerodynamic drag within viscously spreading gas, and how the disc has only a finite reservoir of solids with which to build planets. Prescriptions for pebble accretion were taken from Ormel \& Klahr (2010) and modified for gas turbulence, while those for gas accretion onto cores were drawn from Lee \& Chiang $(2015,2016)$.

A fixed pebble size of $0.01-1 \mathrm{~cm}$ was assumed, motivated by millimeter-wave disc observations that probe these very size particles, and by order-of-magnitude considerations of the limit to which particles can grow by sticking (e.g. Chiang \& Youdin 2010, their section 4). In what follows, we will present some auxiliary calculations that relax this assumption and utilize more sophisticated grain growth prescriptions.

We summarize our results as follows, placing them into context with observations:

(i) Growth by pebble accretion is exponentially sensitive to solid disc mass. The solid disc mass controls not only how massive a core can grow, but also how fast it grows. That growth is at least exponentially fast during the earliest stages if not the entire duration of pebble accretion, with an e-folding time that scales inversely with the disc solid surface density. Growth can be super-exponentially fast if outer disc solids 'pile up' at the position of the core as they drift inward.

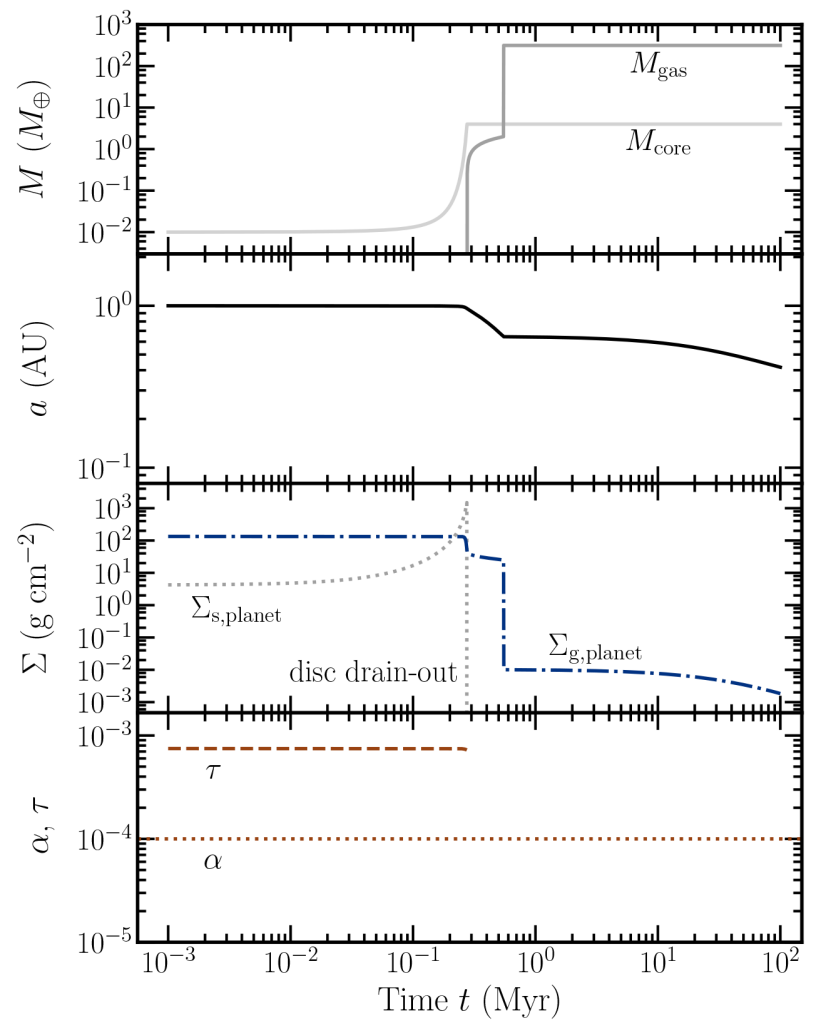

Figure 8. Genesis of a warm Jupiter. All quantities are evaluated at the location of the planet. The core mass $M_{\text {core }}$ grows most rapidly when the local solid surface density $\Sigma_{\mathrm{s} \text {, planet }}$ rises from a particle pile-up (compare first and third panels from the top). This pile-up just precedes the drain-out of solids from outside the core's orbit (see, e.g. Fig. 2). Dips in $\Sigma_{\mathrm{g} \text {, planet }}$ reflect the deepening of gaps in the gas disc following planet mass growth. According to our toy prescription for runaway gas accretion, when the planet's gas mass $M_{\text {gas }}=0.5 M_{\text {core }}$, we instantly set the total planet mass $M_{\text {planet }}=M_{\text {gas }}+M_{\text {core }}=1 M_{\mathrm{J}}$. The planet has technically not stopped migrating at the end of the simulation, but we expect it to eventually park not too far away, given the steady decline in $\Sigma_{\mathrm{g} \text {, planet }}$ from viscous diffusion onto the star (the actual migration history will depend on the actual dispersal history of the disc which is beyond the scope of this paper). Input parameters for the model shown are: $\alpha=10^{-4}, s=0.1 \mathrm{~cm}, a_{1}=100 \mathrm{au}, a(0)=1 \mathrm{au}$, $M_{\text {disc, },}=10 M_{\mathrm{J}}, M_{\mathrm{disc}, \mathrm{s}}=100 M_{\oplus}$.

This strong sensitivity of core growth to disc solid content (see, e.g. Bitsch et al. 2015a,b and Lambrechts \& Johansen 2014 for the same qualitative point), coupled with the need for Jupiters to nucleate from sufficiently massive cores, accords with the observation that the occurrence rates of gas giants (Fischer \& Valenti 2005) and of larger planets more generally (Buchhave et al. 2014; Petigura et al. 2018) correlate with host star metallicity (to the extent that the latter can be used as a proxy for solid disc content). Although the expectation that discs with more solids spawn more massive cores may seem obvious, and is not specific to pebble accretion but also characterizes core formation by giant impacts (Dawson, Chiang \& Lee 2015), it is not a universal prediction of theory - not even in the context of pebble accretion. For example, if the final core mass were limited instead by the pebble isolation mass (e.g. Lambrechts, Johansen \& Morbidelli 2014), a correlation between planet radius and host star metallicity would not be expected, as the pebble isolation mass has no dependence on disc solid mass (see equation 27). In our simulations, pebble isolation is not an issue unless the core migrates inside $\sim 0.1 \mathrm{au}$; what typically limits the core mass instead 


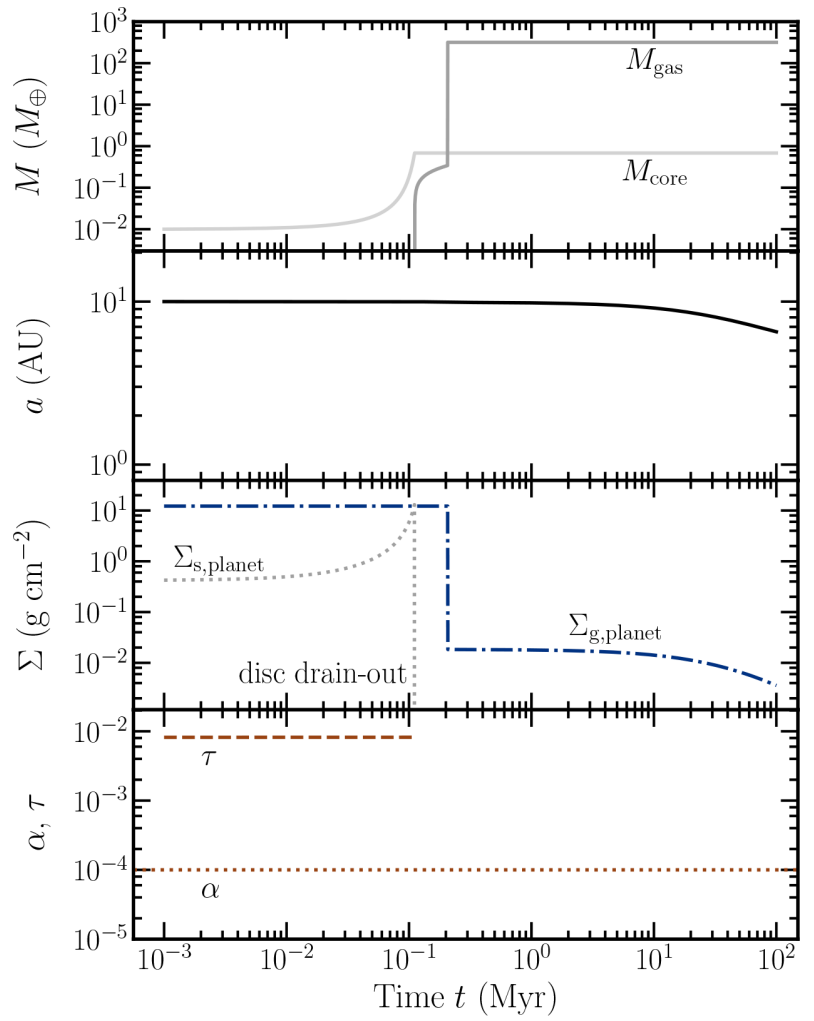

Figure 9. Genesis of a cold Jupiter. Caption text for Fig. 8 applies here. Input parameters: $\alpha=10^{-4}, s=0.1 \mathrm{~cm}, a_{1}=100 \mathrm{au}, a(0)=10 \mathrm{au}$, $M_{\text {disc }, \mathrm{g}}=10 M_{\mathrm{J}}, M_{\mathrm{disc}, \mathrm{s}}=100 M_{\oplus}$.

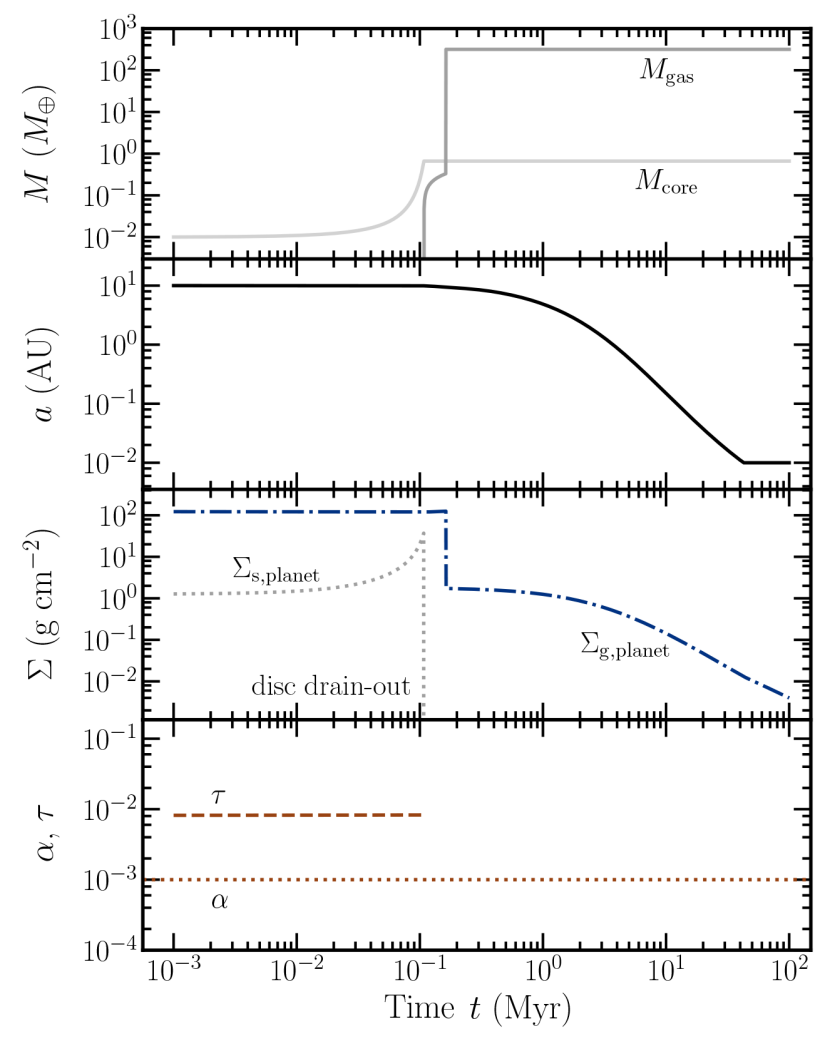

Figure 10. Genesis of a hot Jupiter. Caption text for Fig. 8 applies here. Input parameters: $\alpha=10^{-3}, s=1 \mathrm{~cm}, a_{1}=100 \mathrm{au}, a(0)=10 \mathrm{au}, M_{\text {disc, } \mathrm{g}}=$ $100 M_{\mathrm{J}}, M_{\mathrm{disc}, \mathrm{s}}=300 M_{\oplus}$.

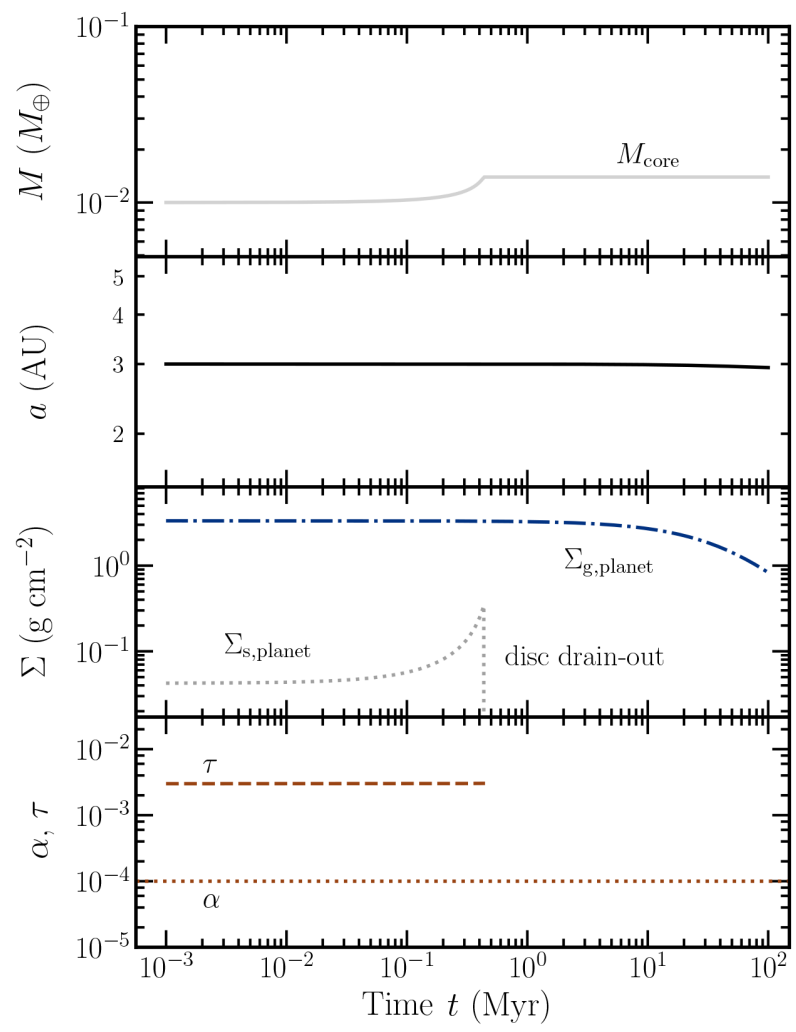

Figure 11. A case where pebble accretion fails to grow much of anything. Although $\tau>\alpha$ (see Fig. 7), the total inventory of solids $M_{\text {disc, s }}$ is too small. Plot format follows that of Fig. 8. Input parameters: $\alpha=10^{-4}, s=0.01 \mathrm{~cm}$, $a_{1}=100 \mathrm{au}, a(0)=30 \mathrm{au}, M_{\mathrm{disc}, \mathrm{g}}=10 M_{\mathrm{J}}, M_{\mathrm{disc}, \mathrm{s}}=30 M_{\oplus}$.

is the fact that the disc only has so much mass to give before it drifts past the core.

(ii) Pebble accretion depends sensitively on particle size and turbulent vertical stirring, and loses at least 1-2 orders of magnitude more mass to radial drift than is actually used to build cores. Forming cores massive enough to nucleate gas giants (i.e. Earth-mass or larger objects) places stringent constraints on the parent disc. Not only must the disc contain enough solids $\left(M_{\mathrm{disc}, \mathrm{s}}>30 M_{\oplus}\right)$, but those solids should have aerodynamic stopping times not too short, and be embedded in gas that is not too turbulent. More quantitatively, over much of our parameter space, the final core mass increases exponentially with $\tau / \alpha$ raised to some power (equations (37) and (38)), with $\tau \equiv \Omega t_{\text {stop }}<1$ the dimensionless measure of particle stopping time, $\Omega$ the orbital angular frequency, and $\alpha<1$ the turbulent Mach number. As $\tau$ increases up to unity, the accretion cross-section of the core grows; as $\alpha$ decreases, the vertical thickness of the solid disc decreases and the density of accreting particles increases. We find empirically that Jupiter-breeding cores require $\tau / \alpha>0.1$. Even when this condition is satisfied - and in this regard, the arguments by Pinte et al. (2016), Fung \& Chiang (2017), and Fung \& Lee (2018) for low $\alpha$, practically inviscid discs are encouraging - pebble accretion is still wasteful in the sense that at least $\sim 90$ per cent, and possibly much more of the solids can be lost to radial drift while growing a single core from a lunar mass to a few Earth masses (see also Ormel 2017, his section 7.1.4 and references therein). In our models, the accretion efficiency of a single core may be $\sim 1-$ 3 per cent (cf. Figs. 6, 15, and 16; see also Guillot, Ida \& Ormel 2014; Lambrechts \& Johansen 2014; Ida, Guillot \& Morbidelli 2016; Picogna, Stoll \& Kley 2018). This single-core efficiency is 


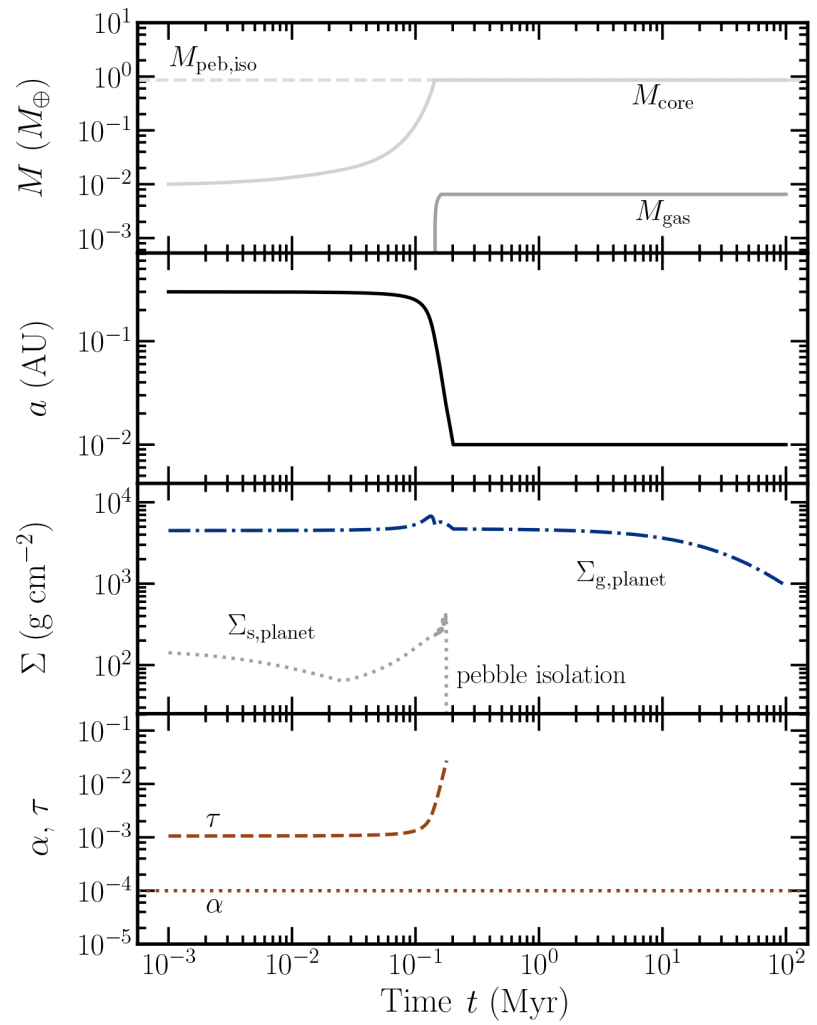

Figure 12. A case where pebble accretion forms a hot Earth but not a Jupiter. Although $\tau>\alpha$ and $M_{\text {disc, } \mathrm{s}}$ is assigned its highest possible value, a gas giant fails to form because the planet's orbital radius starts small and only gets smaller by migration. Small orbits are more susceptible to pebble isolation $\left(M_{\text {peb, iso }} \propto a^{3 / 4}\right.$; see equations (27) and (5)), which is what ultimately limits the core mass here. Gas accretion is quickly terminated once the atmospheric mass reaches the upper bound appropriate to an isothermal atmosphere - an upper bound made low by the high temperature $(2600 \mathrm{~K})$ at the innermost disc edge. Plot format follows that of Fig. 8. Input parameters: $\alpha=10^{-4}$, $s=1 \mathrm{~cm}, a_{1}=100 \mathrm{au}, a(0)=0.3 \mathrm{au}, M_{\mathrm{disc}, \mathrm{g}}=100 M_{\mathrm{J}}, M_{\mathrm{disc}, \mathrm{s}}=1000 M_{\oplus}$.

relevant for deciding whether a given disk has enough solids to generate even a single core massive enough to spawn a gas giant.

(iii) Sub-Earths and gas giants, but no super-Earths - at least none that avoid wholesale migration to the innermost disc edge. Pebble accretion seems to be an all-or-nothing (and usually nothing) prospect: either sub-Earth cores remain sub-Earth, growing by less than a factor of 10 in mass, or conditions are tuned such that cores grow rapidly while the disc is still gas-rich, leading to Jupiters. The exponential dependence of core mass on disc properties (equation (36)) acts effectively as an on/off switch. When the switch is on, and cores grow to maximum, typically super-Earth size, they do not remain super-Earths, but run away to become Jupiters under the early-stage, gas-rich conditions presumed by pebble accretion.

These results are robust against our assumption of a fixed particle size. More realistic grain growth models find that fragmentation of particle aggregates limits Stokes numbers $\tau \lesssim 0.1$ (larger $\tau$ leads to faster and more destructive particle collisions; e.g. Birnstiel, Klahr $\&$ Ercolano 2012, their fig. 6). If we assume instead that particles have a fixed Stokes number $\tau \in\{0.01,0.1\}$, then we find the same general outcome: sub-Earths, Jupiters, and wholly migrated superEarths, as seen in Fig. 14. Comparison with Fig. 5 reveals that this alternative assumption of constant $\tau$ produces a short-period pileup of planets that extends to somewhat larger core masses (up to

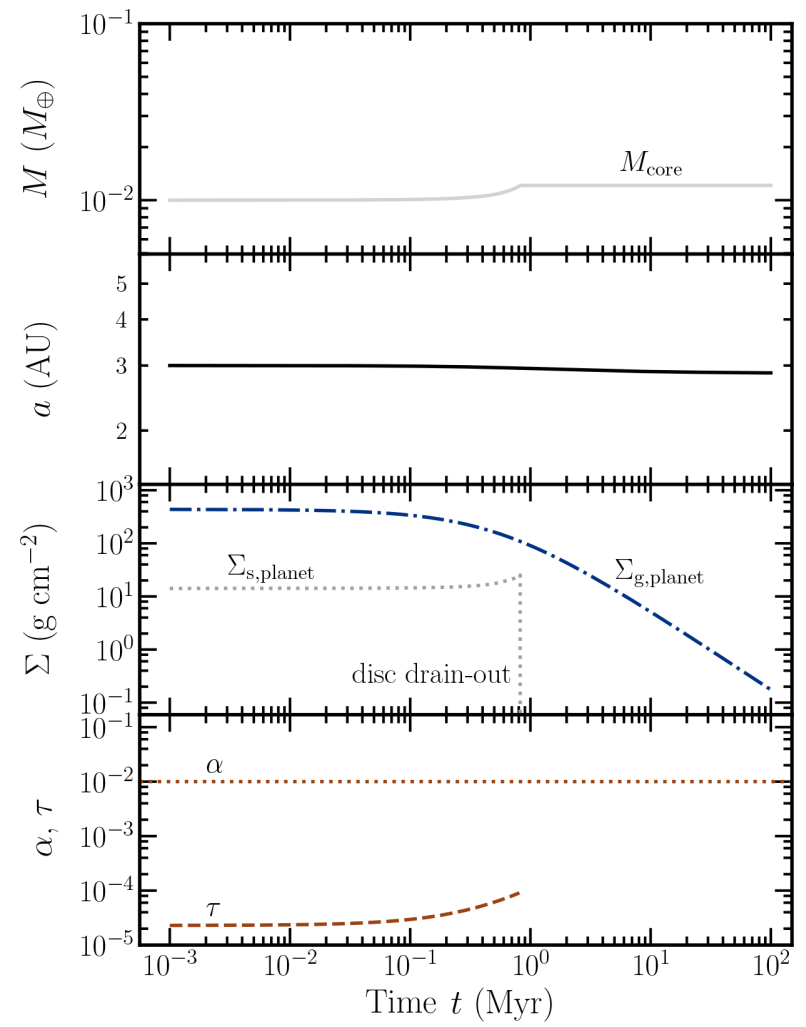

Figure 13. A case where pebble accretion is rendered practically impotent, here because $\tau \ll \alpha$; particles are too strongly coupled to gas to drop out onto cores, and particle densities are made too dilute by turbulent stirring. Furthermore, because $\tau \ll \alpha$, the particle pile-up at the planet's position is more muted than in other figures in this series. Plot format follows that of Fig. 8. Input parameters: $\alpha=10^{-2}, s=0.01 \mathrm{~cm}, a_{1}=100 \mathrm{au}, a(0)=3 \mathrm{au}$, $M_{\text {disc, },}=100 M_{\mathrm{J}}, M_{\mathrm{disc}, \mathrm{s}}=1000 M_{\oplus}$.

py

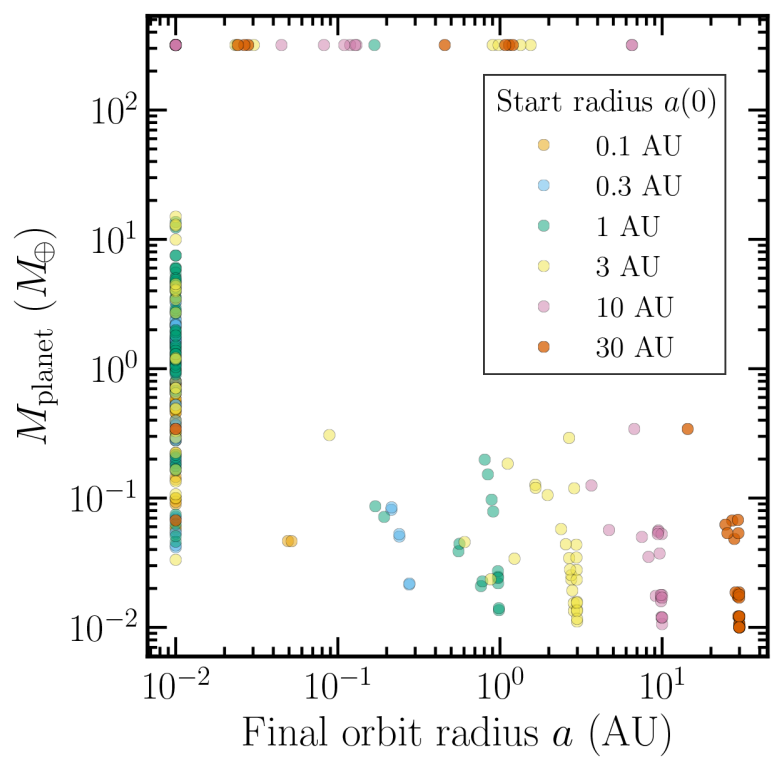

Figure 14. Same as Fig. 5, but for fixed Stokes number $\tau \in\{0.01,0.1\}$, in lieu of fixed particle size $s \in\{0.01,0.1,1\} \mathrm{cm}$. All models shown have a solid drift time-scale, $a_{1} /\left[v_{\text {drift }}\left(a_{1}, 0\right)\right]$, shorter than $1 \mathrm{Myr}$ (i.e. there are no squares, only circles, unlike in Fig. 5). 
$\sim 10-20 M_{\oplus}$ ), and discs that drain even faster (all discs have drift time-scales $<1$ Myr).

Runaway can be avoided at ultra-small orbital distances where temperatures are high enough to stop gas accretion. Those superEarths $\left(1-10 M_{\oplus}\right)$ that do form in our models are all located at the innermost disc edge $(0.01 \mathrm{au})$, having migrated and piled up there. The problem is that short-period pile-ups of planets are not observed (Lee \& Chiang 2017); nor is it clear how to disrupt the mean-motion resonant chains that may result from such wholesale migration, in sufficient proportions to match observations (Izidoro et al. 2017; but see Goldreich \& Schlichting 2014). If migration were somehow suppressed, super-Earths could be formed at a variety of orbital distances, as we have verified by direct experimentation. Short of finding a mechanism to shut off migration (but see Fung \& Chiang 2017 and Fung \& Lee 2018 for thoughts along these lines), we submit that super-Earths are more naturally created later in a disc's life, under gas-poor conditions where migration is not a concern, in a series of late-stage giant impacts (e.g. Lee et al. 2014; Dawson et al. 2015; Dawson, Lee \& Chiang 2016; Inamdar \& Schlichting 2016; Lee \& Chiang 2016; Ogihara et al. 2018).

The gas giants that form by pebble accretion also generally undergo orbital migration. This is a simple consequence of the gas-rich conditions typically assumed by pebble accretion. The extent of migration exhibited by our model Jupiters ranges from shrinking the orbital radius by $\sim 30$ per cent, to complete collapse from 10 au to the innermost disc edge - and everything in between. Had we adopted a more realistic (i.e. slower) prescription of gas accretion during the runaway phase (see Machida et al. 2010 and Bitsch et al. 2015b), our model Jupiters would have migrated farther, or been transformed into sub-Saturns - planets larger than Neptune but smaller than Saturn. Contrary to the speculation that hot Jupiter cores can form in situ by pebble accretion (Batygin, Bodenheimer \& Laughlin 2016), we find that pebble isolation inside $\sim 0.1$ au limits core masses to values too low to trigger runaway gas accretion within the gas disc lifetime.

(iv) Jupiters can form by pebble accretion, but in discs that may not fit mm-wave observations. Pebble accretion favours particles large enough to have long stopping times $\tau$ (up to unity). The problem is that such particles are also the fastest to drain out of the disc. In nearly all of our Jupiter-producing runs, the disc is emptied of $0.01-1 \mathrm{~cm}$ sized pebbles on time-scales ranging from 0.01 to $1 \mathrm{Myr}$. These drift times are troublingly shorter than the $1-$ $10 \mathrm{Myr}$ ages of discs seen in thermal mm-wave emission on scales of $10-100 \mathrm{au}$.

Our findings, in particular our conclusions about the extreme sensitivity of pebble accretion rates to disc parameters, highlight perhaps under-appreciated difficulties in forming gas giants - and the apparent impossibility of forming super-Earths outside the innermost disc edge - by pebble accretion. For example, Lambrechts \& Johansen (2014, LJ14) reported that cores with initial masses of $10^{-3} M_{\oplus}$ readily grow by pebble accretion to $1-10 M_{\oplus}$ within $1 \mathrm{Myr}$ at orbital distances of 5-20 au. Their result follows from assuming that (a) pebble accretion proceeds in the settling limit ( $\tau \lesssim 1$ ), (b) the pebbles effectively comprise a $2 \mathrm{D}$ ultra-dense sheet whose scale height $H_{\mathrm{s}}$ is less than the accretion impact parameter $R_{\text {acc }}$, and (c) Keplerian shear dominates the headwind in setting the relative velocity between pebble and core (cf. our equation (14)). Invoking these assumptions gives the largest possible growth rates, with maximal accretion cross-sections and velocities set by Hillsphere scales (their equation (28)). In Fig. 15, we compare their optimistic solutions against other solutions that do not make the

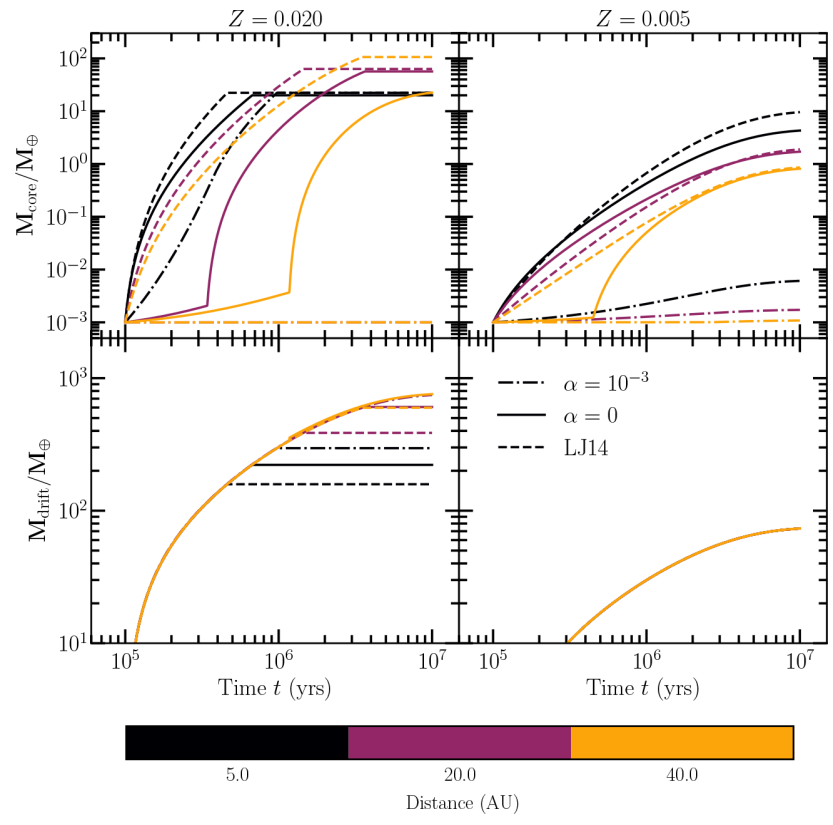

Figure 15. Comparison between the pebble accretion solution of Lambrechts \& Johansen (2014, LJ14, dashed) and solutions that explicitly account for $\alpha$. To isolate the dependence on $\alpha$, we use the same input parameters as LJ14 - in particular, we incorporate their grain growth prescriptions, as outlined in Section 2 of LJ14 (their equations (20) and (25)); their initial core mass of $10^{-3} M_{\oplus}$; and their fiducial gas surface density $\Sigma_{\mathrm{g}}=500 \mathrm{~g} \mathrm{~cm}^{-2}(\mathrm{a} / \mathrm{au})^{-1} \exp (-t / 3 \mathrm{Myr})$. For $\alpha=10^{-3}$ (dot-dashed), accretion is not $2 \mathrm{D}\left(R_{\mathrm{acc}}<H_{\mathrm{s}}\right)$ and is therefore less efficient than in the LJ14 solution. For $\alpha=0$ (solid), accretion is $2 \mathrm{D}$, as was assumed by $\mathrm{LJ} 14$, but accretion is not always in the settling regime, contrary to the assumption of LJ14. Top: Core mass vs. time. Core growth is truncated once the pebble isolation mass is reached $\left(20 M_{\oplus}(a / 5 \mathrm{au})^{3 / 4}\right.$, equation (34) of LJ14). The assumption of shear-dominated, settling accretion is seen to overestimate core growth rates at large distances and early times. At 40 au, cores hardly grow for $\sim 1 \mathrm{Myr}$, especially for a disc-integrated, initial solid-to-gas mass ratio $Z$ ( $=Z_{0}$ in the notation of $\left.\mathrm{LJ} 14\right)$ of 0.02 . In the grain growth model of $\mathrm{LJ} 14$, the higher $Z$ is, the more rapidly particles grow, and the larger is $\tau$; for $Z=0.02$, the stopping time is longer than the core-particle encounter time at $a=40$ au for the first $\sim 1 \mathrm{Myr}$, and pebble accretion is in the (slow) hyperbolic regime. Bottom: The cumulative 'wasted' mass that drifts past the core as the core is growing. All model curves necessarily overlap except when the core in a given model stops growing. Comparing our solutions in the top panel with our solutions in the bottom panel, we infer net pebble accretion efficiencies that are at most $M_{\text {core }} / M_{\text {drift }} \simeq 1 / 10(Z=0.02, a=5 \mathrm{au}$, $\alpha=0)$.

same assumptions. We focus on the sensitivity of the evolution to $\alpha$, setting other parameters such as the gas surface density, initial core mass, and pebble size (see below for more comments related to particle size) to the same values used by LJ14. For $\alpha=10^{-3}$, we see that accretion rates can be overestimated by orders of magnitude. Even if we assume that $\alpha=0$ to strictly enforce 2D accretion, core growth can be delayed significantly, as accretion begins and persists in the hyperbolic regime for up to 1 Myr at $a \sim 20-40 \mathrm{au}$. Since such a delay is comparable to the gas disk lifetime, cores built by pebble accretion at large distances may not be able to nucleate gas giants, unless disk conditions are finely tuned or seed cores are more massive (cf. Fig. 16).

Note further that to ensure the comparison in Fig. 15 is fair, we incorporated LJ14's scheme for grain growth (their equations (20) and (25); see also Birnstiel et al. 2012). Their model disc is characterized by particle stopping times $\tau \sim 0.01-0.1$ (their fig. 1); 


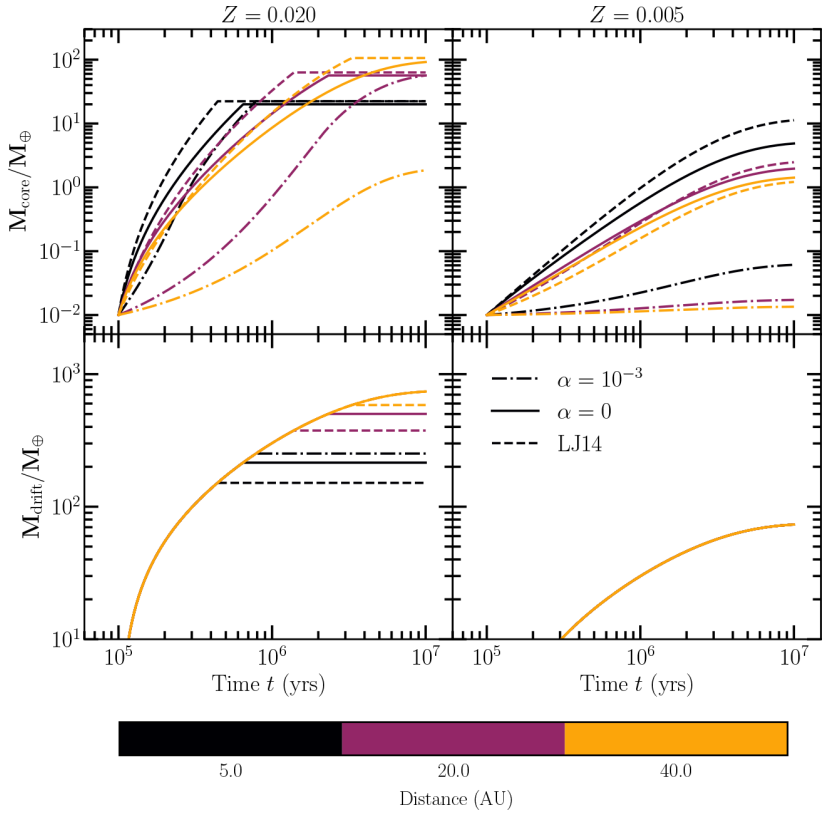

Figure 16. Same as Fig. 15 but starting with a larger seed core of $0.01 M_{\oplus}$, the mass at which pebble accretion might begin in earnest (see Johansen \& Lambrechts 2017, their fig. 8). With a larger starting mass, there is better agreement between the explicit $\alpha$ models and LJ14, although at $\alpha-10^{-3}$ growth is still slow at $a=40$ au.

these are relatively high values that on the one hand promote pebble accretion, but on the other lead to fast radial drift and therefore disc sizes too compact compared to mm-continuum observations. Fig. 17 shows that the LJ14 model of grain growth + drift (solid lines) does not yield enough mm-cm objects at 20-60 au to match the size-wavelength relation exhibited by TW Hya, a 3-10 Myr system. Our models suffer from the same problem, as we have noted under (iv) above.

Powell, Murray-Clay \& Schlichting (2017) solved the problem of reproducing the size-wavelength relation of TW Hya by enhancing the disc's gas mass and slowing drift. This seems to us an acceptable solution. Our comment would be that such a disc would not be conducive to pebble accretion of mm-cm-sized particles whose $\tau$ 's would be too small to accrete onto cores efficiently. Perhaps cores grow instead from super-cm (larger $\tau$ ) particles that are less visible to $\mathrm{mm}-$ continuum observations. Another way to explain the sizewavelength correlation is to invoke optically thick substructures in the inner disc; these could take the form of concentric rings of particles trapped in gas pressure maxima (see Tripathi et al. 2018 for a specific discussion of UZ Tau, and also our Section 4.1 below).

Our conclusions do not seem particularly sensitive to our assumption of a single seed core. Were we to distribute seed cores over a wide range of orbital distances, those close in would grow more-or-less independently of those far out, since any given core diverts only a small fraction - a few per cent at most - of the background disc flow towards its own growth. If seed cores were packed so closely as to be competing for the same solids, and if $R_{\mathrm{acc}}<H_{\mathrm{s}}$, then growth would be 'neutral' (section 8 of Goldreich et al. 2004): the distribution of relative core masses would not change, since the doubling time $M_{\text {core }} / \dot{M}_{\text {core }} \propto M_{\text {core }}^{0}$ (equation (35)). If $R_{\text {acc }}>H_{\mathrm{s}}$, then growth would be 'orderly': $M_{\text {core }} / \dot{M}_{\text {core }}$ would increase with increasing $M_{\text {core }}$ (equations (A6) and (A8)), and the distribution of relative core masses would narrow (Kretke \& Levison 2014).

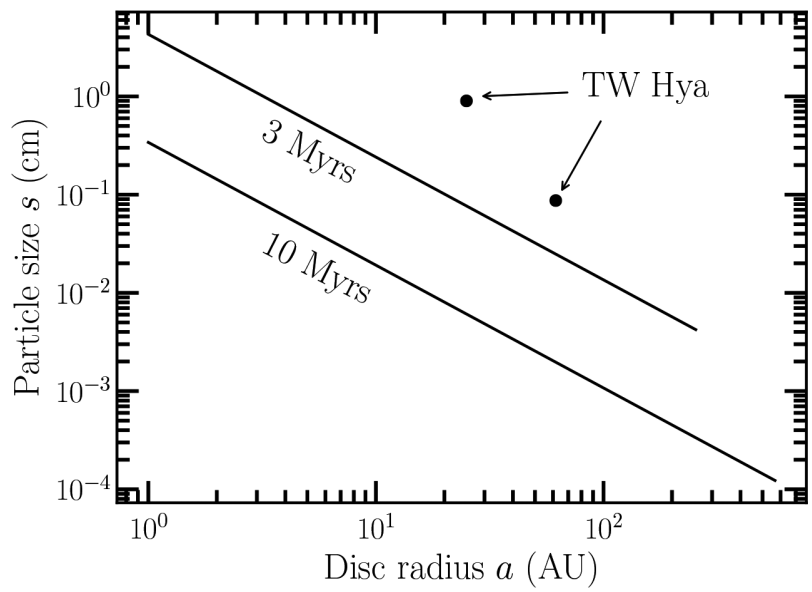

Figure 17. Comparing the grain growth model of Lambrechts \& Johansen (2014, LJ14, solid lines) to the observed mm-cm continuum sizes of the 3-10 Myr old TW Hydra disc (Andrews et al. 2012; Menu et al. 2014; black circles). The latter data are placed on this plot by assuming that the size of the emitting particles equals the wavelength of observation. The fiducial model of LJ14 predicts insufficiently large particles at 20-100 au at an age of 3-10 Myr; by this time, $\mathrm{mm}$-cm-sized objects have drained out by gas drag. Staying within the framework of the LJ14 grain growth model, this discrepancy can be resolved by assuming a longer-lived gas disc (one with an e-folding time of $10 \mathrm{Myr}$ ), and a disc-integrated dust-to-gas ratio that is strongly supersolar $(Z=0.1)$ to enhance grain growth. An alternative (still within the context of the LJ14 model) is to make the disc so gas-heavy (approximately 10 times more massive than their fiducial disc) that it is Toomre $Q$-unstable ( $Q \sim 0.4$ at $a=25$ au). We note in passing that using the model disc of Bitsch et al. (2015a,b), which is approximately three times more dense than the fiducial LJ14 disc beyond $\sim 10$ au, produces model curves that approach but still do not match the observed data for TW Hya.

\subsection{Future directions}

Our model could use improvement in many respects. There are, of course, the usual shortcomings resulting from our incomplete understanding of orbital migration (e.g. the transition from Type I to Type II); runaway gas accretion; and perhaps most glaringly, disc turbulence and transport. We focus here on issues more specific to pebble accretion.

Revisiting the gas-particle dynamics of pebble accretion from first principles seems worthwhile. Our paper is based on the equations of Ormel \& Klahr (2010), which assume a strict Cartesian shear for the background gas disc. But cores perturb gas streamlines onto horseshoe orbits in 2D (e.g. Ormel 2013, their fig. 12) and 'transient horseshoes' in 3D (e.g. Fung, Artymowicz \& Wu 2015), either of which can deflect particles, particularly those with small $\tau$, away from the core, and conceivably radically altering accretion probabilities. Xu, Bai \& Murray-Clay (2017) have tested some of the scaling relations of Ormel \& Klahr (2010) using 3D simulations, but only under restrictive conditions and with mixed results. A more comprehensive study, starting with re-deriving accretion cross-sections and velocities for 2D laminar flow patterns, would be welcome (see also Popovas et al. 2018).

Images of protoplanetary discs from the Atacama Large Millimeter Array have revealed concentric rings of dust (ALMA Partnership et al. 2015; Andrews et al. 2016; S. Andrews 2017, personal communication). These rings may trace local gas pressure maxima that can 'trap' inflowing pebbles (see the review by Pinilla \& Youdin 2017 and references therein). Are these rings the sites of planetesimal/planet formation? How does pebble accretion proceed in the 
presence of traps? In particular, how would the accretion rate (35) and the efficiency (40) change if the seed core were located at the centre of the pressure bump? Because drift speeds slow to zero in traps, interparticle collisions are gentler, and bodies may stick their way to larger sizes and larger Stokes numbers (cf. Chiang \& Youdin 2010, their section 4), allowing for more rapid pebble accretion. How long the solids in the trap take to congeal into a single body is an outstanding question.

\section{ACKNOWLEDGEMENTS}

We thank Bertram Bitsch, Jeffrey Fung, Anders Johansen, Michiel Lambrechts, Wladimir Lyra, Ruth Murray-Clay, Chris Ormel, Diana Powell, Michael Rosenthal, Julia Venturini, and an anonymous referee for comments that helped to improve the manuscript. JWL and EC acknowledge support from the National Science Foundation and a Berkeley Excellence Accounts for Ressearch (BEAR) grant. EJL is supported by the Sherman Fairchild Fellowship from Caltech. This research used the Savio computational cluster resource provided by the Berkeley Research Computing program at the University of California, Berkeley (supported by the UC Berkeley Chancellor, Vice Chancellor for Research, and Chief Information Officer).

\section{REFERENCES}

ALMA Partnership et al., 2015, ApJ, 808, L3

Ali-Dib M., Johansen A., Huang C. X., 2017, MNRAS, 469, 5016

Andrews S. M. et al., 2012, ApJ, 744, 162

Andrews S. M. et al., 2016, ApJ, 820, L40

Ataiee S., Baruteau C., Alibert Y., Benz W., 2018, A\&A, 615, A110

Batygin K., Bodenheimer P. H., Laughlin G. P., 2016, ApJ, 829, 114

Birnstiel T., Klahr H., Ercolano B., 2012, A\&A, 539, A148

Bitsch B., Johansen A., Lambrechts M., Morbidelli A., 2015a, A\&A, 575, A28

Bitsch B., Lambrechts M., Johansen A., 2015b, A\&A, 582, A112

Bitsch B., Morbidelli A., Johansen A., Lega E., Lambrechts M., Crida A., 2018, A\&A, 612, A30

Brauer F., Dullemond C. P., Johansen A., Henning T., Klahr H., Natta A., 2007, A\&A, 469, 1169

Brouwers M. G., Vazan A., Ormel C. W., 2018, A\&A, 611, A65

Buchhave L. A. et al., 2014, Nature, 509, 593

Chiang E., Laughlin G., 2013, MNRAS, 431, 3444

Chiang E., Youdin A. N., 2010, Annu. Rev. Earth Planet. Sci., 38, 493

D’Angelo G., Lubow S. H., 2010, ApJ, 724, 730

Dawson R. I., Chiang E., Lee E. J., 2015, MNRAS, 453, 1471

Dawson R. I., Lee E. J., Chiang E., 2016, ApJ, 822, 54

Dipierro G., Laibe G., 2017, MNRAS, 469, 1932

Dong R., Fung J., 2017, ApJ, 835, 146

Duffell P. C., Haiman Z., MacFadyen A. I., D’Orazio D. J., Farris B. D., 2014, ApJ, 792, L10

Fischer D. A., Valenti J., 2005, ApJ, 622, 1102

Fung J., Chiang E., 2017, ApJ, 839, 100

Fung J., Lee E. J., 2018, ApJ, 859, 126

Fung J., Shi J.-M., Chiang E., 2014, ApJ, 782, 88

Fung J., Artymowicz P., Wu Y., 2015, ApJ, 811, 101

Ginzburg S., Schlichting H. E., Sari R., 2016, ApJ, 825, 29

Goldreich P., Schlichting H. E., 2014, AJ, 147, 32

Goldreich P., Lithwick Y., Sari R., 2004, ARA\&A, 42, 549

Guillot T., Ida S., Ormel C. W., 2014, A\&A, 572, A72

Hartmann L., Calvet N., Gullbring E., D’Alessio P., 1998, ApJ, 495, 385

Ida S., Lin D. N. C., 2008, ApJ, 673, 487
Ida S., Guillot T., Morbidelli A., 2016, A\&A, 591, A72

Inamdar N. K., Schlichting H. E., 2016, ApJ, 817, L13

Izidoro A., Ogihara M., Raymond S. N., Morbidelli A., Pierens A., Bitsch B., Cossou C., Hersant F., 2017, MNRAS, 470, 1750

Johansen A., Lambrechts M., 2017, Annu. Rev. Earth Planet. Sci., 45, 359

Kanagawa K. D., Muto T., Tanaka H., Tanigawa T., Takeuchi T., Tsukagoshi T., Momose M., 2015, ApJ, 806, L15

Kanagawa K. D., Tanaka H., Szuszkiewicz E., 2018, ApJ, 861, 140

Kley W., Nelson R. P., 2012, ARA\&A, 50, 211

Kretke K. A., Levison H. F., 2014, AJ, 148, 109

Lambrechts M., Johansen A., 2012, A\&A, 544, A32

Lambrechts M., Johansen A., 2014, A\&A, 572

Lambrechts M., Lega E., 2017, A\&A, 606, A146

Lambrechts M., Johansen A., Morbidelli A., 2014, A\&A, 572, A35

Lee E. J., Chiang E., 2015, ApJ, 811, 41

Lee E. J., Chiang E., 2016, ApJ, 817

Lee E. J., Chiang E., 2017, ApJ, 842, 40

Lee A. T., Chiang E., Asay-Davis X., Barranco J., 2010a, ApJ, 718, 1367

Lee A. T., Chiang E., Asay-Davis X., Barranco J., 2010b, ApJ, 725, 1938

Lee E. J., Chiang E., Ormel C. W., 2014, ApJ, 797

Levison H. F., Kretke K. A., Duncan M. J., 2015, Nature, 524, 322

Lynden-Bell D., Pringle J. E., 1974, MNRAS, 168, 603

Machida M. N., Kokubo E., Inutsuka S.-I., Matsumoto T., 2010, MNRAS, 405,1227

Menu J. et al., 2014, A\&A, 564, A93

Morbidelli A., Lambrechts M., Jacobson S., Bitsch B., 2015, Icarus, 258, 418

Ogihara M., Kokubo E., Suzuki T. K., Morbidelli A., 2018, A\&A, 615, A63

Ormel C. W., 2013, MNRAS, 428, 3526

Ormel C. W., 2014, ApJ, 789, L18

Ormel C. W., 2017, The Emerging Paradigm of Pebble Accretion. Springer International Publishing, Cham, p. 197

Ormel C. W., Klahr H. H., 2010, A\&A, 520, A43

Ormel C. W., Kobayashi H., 2012, ApJ, 747, 115

Ormel C. W., Liu B., 2018, A\&A, 615, A178

Ormel C. W., Liu B., Schoonenberg D., 2017, A\&A, 604, A1

Perets H. B., Murray-Clay R. A., 2011, ApJ, 733, 56

Pérez L. M. et al., 2015, ApJ, 813, 41

Petigura E. A. et al., 2018, AJ, 155, 89

Picogna G., Stoll M. H. R., Kley W., 2018, preprint (arXiv:1803.08730)

Pinilla P., Youdin A., 2017, Particle Trapping in Protoplanetary Disks: Models vs. Observations. Springer International Publishing, Cham, p. 91-142

Pinte C., Dent W. R. F., Ménard F., Hales A., Hill T., Cortes P., de GregorioMonsalvo I., 2016, ApJ, 816, 25

Piso A.-M. A., Youdin A. N., 2014, ApJ, 786, 21

Pollack J. B., Hubickyj O., Bodenheimer P., Lissauer J. J., Podolak M., Greenzweig Y., 1996, Icarus, 124, 62

Popovas A., Nordlund Å., Ramsey J. P., Ormel C. W., 2018, MNRAS, 479, 5136

Powell D., Murray-Clay R., Schlichting H. E., 2017, ApJ, 840

Rosenthal M. M., Murray-Clay R. A., Perets H. B., Wolansky N., 2018, ApJ, 861, 74

Rosotti G. P., Juhasz A., Booth R. A., Clarke C. J., 2016, MNRAS, 459, 2790

Shakura N. I., Sunyaev R. A., 1973, A\&A, 24, 337

Tazzari M. et al., 2016, A\&A, 588, A53

Tripathi A., Andrews S. M., Birnstiel T., Wilner D. J., 2017, ApJ, 845, 44

Tripathi A. et al., 2018, ApJ, 861, 64

Venturini J., Helled R., 2017, ApJ, 848, 95

Venturini J., Alibert Y., Benz W., Ikoma M., 2015, A\&A, 576, A114

Weidenschilling S. J., 1977, MNRAS, 180, 57

Xu Z., Bai X.-N., Murray-Clay R. A., 2017, ApJ, 847, 52

Youdin A. N., Chiang E. I., 2004, ApJ, 601, 1109

Youdin A. N., Lithwick Y., 2007, Icarus, 192, 588 


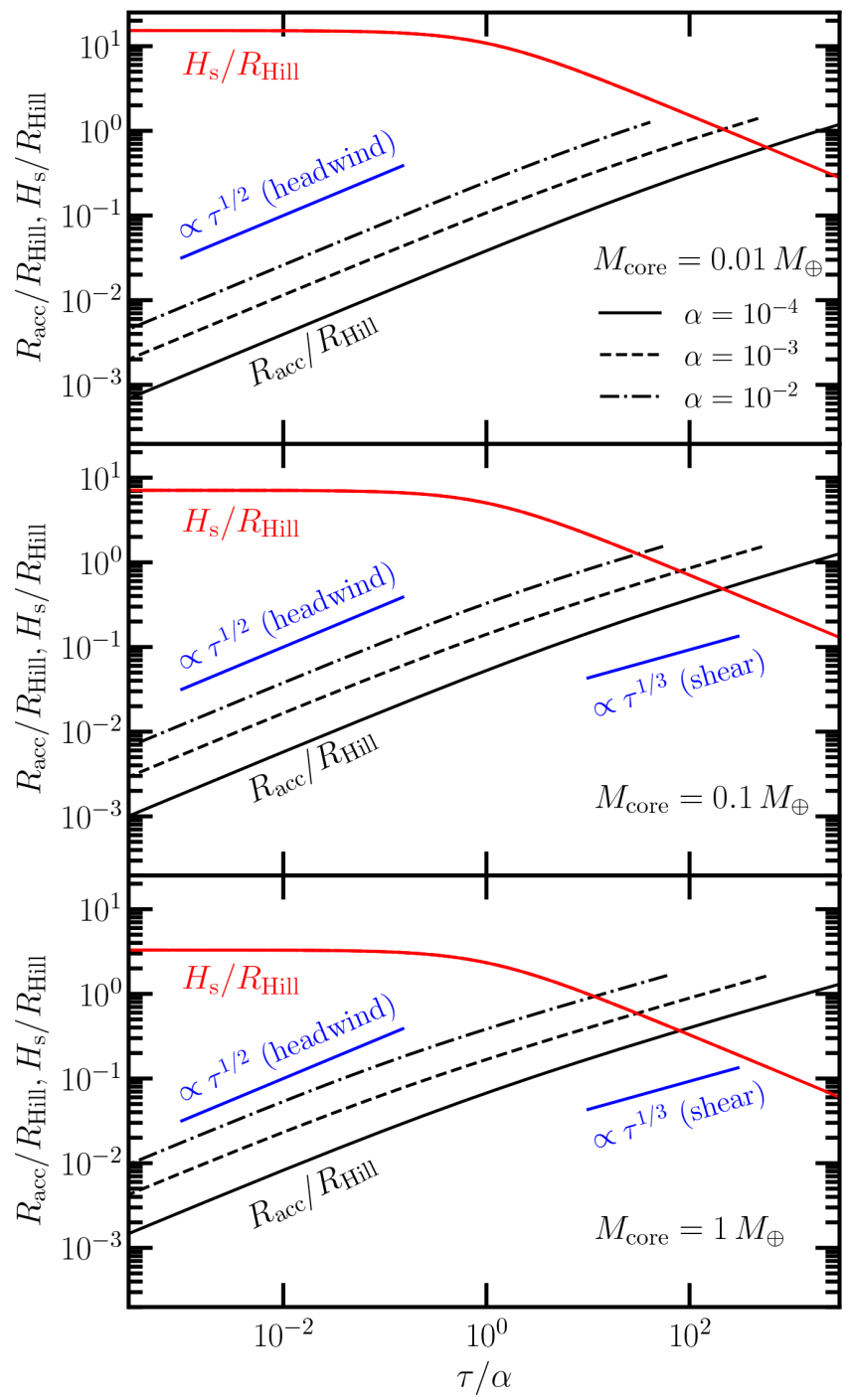

Figure A1. Comparison between the accretion radius $R_{\text {acc }}$ (black lines) and the particle scale height $H_{\mathrm{s}}$ (red lines) as a function of the ratio between particle Stokes number (dimensionless stopping time) $\tau$ and turbulent Mach number $\alpha$. We truncate an $R_{\text {acc }} / R_{\text {Hill }}$ curve when pebble accretion ceases to be in the settling regime (i.e. when equation (15) is not satisfied). All values are calculated at $a=5 \mathrm{au}$. At fixed core mass, accretion is headwinddominated at small $\tau / \alpha$ and shear-dominated at large $\tau / \alpha$ (compare $R_{\text {acc }} / R_{\text {Hill }}$ curves to blue segments; see equations (A1) and (A3)).

\section{APPENDIX: REGIMES OF CORE GROWTH BY PEBBLE ACCRETION}

Over much of our parameter space, the accretion radius $R_{\mathrm{acc}}$ is smaller than the particle scale height $H_{\mathrm{s}}$. For a small subset of our models, at core masses above a few $M_{\oplus}$, this inequality reverses (see Fig. A1; to be clear, the equations we solve are general enough to accommodate this possibility). We discuss here how the final core mass changes its scaling behaviour with time and other variables across parameter space (cf. equations (35)-(38)), assuming throughout that pebble accretion is in the settling regime (stopping time $\tau \lesssim 1$ ). See Section 2.3.1 for more background.

When the headwind parameter $\zeta=v_{\text {hw }} / v_{\text {Hill }}$ exceeds the accretion radius $b=R_{\text {acc }} / R_{\mathrm{Hill}}$, the accretion velocity is dominated by the headwind velocity: $v_{\text {acc }} \sim v_{\text {hw }}$, which for our disc temperature profile is constant. The accretion radius $R_{\text {acc }}$ follows from equating the kick velocity $\Delta v$ (equation (16)) to $v_{\text {acc }} / 4$ (OK10):

$$
\begin{aligned}
\frac{G M_{\text {core }}}{R_{\text {acc,hw }}^{2}} \frac{\tau}{\Omega} & \sim \frac{v_{\text {hw }}}{4} \\
R_{\text {acc,hw }} & \sim\left(\frac{4 G M_{\text {core }} \tau}{\Omega v_{\text {hw }}}\right)^{1 / 2} .
\end{aligned}
$$

This is equivalent to the wind-shearing radius of Perets \& MurrayClay (2011). Under headwind-dominated conditions, $R_{\mathrm{acc}, \mathrm{hw}}$ exceeds the particle scale height $H_{\mathrm{s}}=H \sqrt{\alpha /(\alpha+\tau)}$ when

$$
\begin{aligned}
\frac{\tau}{\alpha} & >\left(\frac{f_{P}}{4 \alpha}\right)^{1 / 2}\left(\frac{c_{\mathrm{s}}}{\Omega a}\right)^{2}\left(\frac{M_{\star}}{M_{\text {core }}}\right)^{1 / 2} \\
& \gtrsim 100\left(\frac{a}{1 \mathrm{au}}\right)^{1 / 2}\left(\frac{0.01 M_{\oplus}}{M_{\text {core }}}\right)^{1 / 2}\left(\frac{0.001}{\alpha}\right)^{1 / 2}
\end{aligned}
$$

where the second inequality follows from our disc parameters: $f_{P}=11 / 8, T=260 \mathrm{~K}(a / 1 \mathrm{au})^{-1 / 2}$, and $M_{\star}=\mathrm{M}_{\odot}$. Technically the above criterion for whether $R_{\text {acc }}$ exceeds $H_{\mathrm{s}}$ is derived assuming $\tau>\alpha$, but this is a safe assumption; if $\tau<\alpha$, so that small particles are strongly stirred by gas turbulence, then only super-massive cores $\left(\gtrsim 100 M_{\oplus}\right)$ have accretion radii $R_{\text {acc }}>H_{\mathrm{s}}$.

On the other hand, when $\zeta<b$, the accretion velocity is dominated by the Keplerian shearing velocity $v_{\text {acc }} \sim 3 \Omega R_{\text {acc }} / 2$. Then

$$
\begin{aligned}
\frac{G M_{\text {core }}}{R_{\mathrm{acc}, \mathrm{sh}}} \frac{\tau}{\Omega} & \sim \frac{3 \Omega R_{\mathrm{acc}, \mathrm{sh}}}{8} \\
R_{\mathrm{acc}, \mathrm{sh}} & \sim\left(\frac{8 G M_{\mathrm{core}} \tau}{3 \Omega^{2}}\right)^{1 / 3} .
\end{aligned}
$$

The transition from headwind-dominated to shear-dominated accretion (what Lambrechts \& Johansen 2012 call drift accretion versus Hill accretion) occurs when $R_{\mathrm{acc}, \mathrm{hw}} \sim R_{\mathrm{acc}, \mathrm{sh}}$ :

$$
\begin{aligned}
M_{\text {core,trans }} & \sim v_{\mathrm{hw}}^{3} /(9 G \Omega \tau) \\
& \sim 0.1 M_{\oplus}\left(\frac{0.001}{\tau}\right)\left(\frac{a}{1 \mathrm{au}}\right)^{3 / 2} .
\end{aligned}
$$

We emphasize that the transition core mass depends on $\tau$ (see also equation (7.9) of Ormel 2017). Under shear-dominated conditions, $R_{\text {acc }}>H_{\mathrm{s}}$ when

$$
\begin{aligned}
\frac{\tau}{\alpha} & >\left(\frac{3}{8 \alpha}\right)^{2 / 5}\left(\frac{c_{\mathrm{s}}}{\Omega a}\right)^{6 / 5}\left(\frac{M_{\star}}{M_{\text {core }}}\right)^{2 / 5} \\
& \gtrsim 30\left(\frac{a}{1 \mathrm{au}}\right)^{3 / 10}\left(\frac{M_{\oplus}}{M_{\text {core }}}\right)^{2 / 5}\left(\frac{0.001}{\alpha}\right)^{2 / 5}
\end{aligned}
$$

where we have again safely assumed $\tau>\alpha$.

Armed with the above relations, we derive the approximate scaling behaviour of the core mass in various regimes. When $R_{\mathrm{acc}}<H_{\mathrm{s}}$, the accretion rate $\dot{M}_{\text {core }}$ is identical between headwind and sheardominated regimes, and is given by equation (35). The accretion rate is the same between these regimes because $\dot{M}_{\text {core }} \propto R_{\text {acc }}^{2} v_{\text {acc }}$ (when $R_{\text {acc }}<H_{\mathrm{s}}$ ), and the combination $R_{\text {acc }}^{2} v_{\text {acc }}$ is given in the settling limit by its defining condition, $G M_{\text {core }} / R_{\text {acc }}^{2} \times \tau / \Omega \sim v_{\text {acc }} / 4$ (Ormel \& Klahr 2010). The end result is that $M_{\text {core }}(t)$ is given by equation (36) and is exponential if not super-exponential in time, depending on how $\Sigma_{\mathrm{s}}$ evolves at the position of the core.

When $R_{\text {acc }}>H_{\mathrm{s}}$, and when accretion is headwind-dominated, $\dot{M}_{\text {core }}=2 \Sigma_{\mathrm{s}} R_{\mathrm{acc}, \mathrm{hw}} v_{\mathrm{hw}}$, which together with (A1) yields

$\dot{M}_{\text {core }}=4\left(\frac{v_{\text {hw }}}{\Omega a}\right)^{1 / 2} \tau^{1 / 2}\left(\frac{M_{\text {core }}}{M_{\star}}\right)^{1 / 2} \Omega \Sigma_{\mathrm{s}} a^{2}$ 
and

$M_{\text {core }}(t)=\left[M_{\text {core }}(0)^{1 / 2}+2\left(\frac{v_{\mathrm{hw}}}{\Omega a}\right)^{1 / 2} \tau^{1 / 2} \frac{\Omega a^{2}}{M_{\star}^{1 / 2}} \int_{0}^{t} \Sigma_{\mathrm{s}} d t\right]^{2}$

where all quantities are evaluated at the core's position. When $R_{\text {acc }}>H_{\mathrm{s}}$ and accretion is shear-dominated, $\dot{M}_{\text {core }}=3 \Sigma_{\mathrm{s}} R_{\mathrm{acc}, \mathrm{sh}}^{2} \Omega$, so that

$\dot{M}_{\text {core }}=(192)^{1 / 3} \tau^{2 / 3}\left(\frac{M_{\text {core }}}{M_{\star}}\right)^{2 / 3} \Omega \Sigma_{\mathrm{s}} a^{2}$ and

$M_{\text {core }}(t)=\left[M_{\text {core }}(0)^{1 / 3}+\left(\frac{8}{3}\right)^{2 / 3} \tau^{2 / 3} \frac{\Omega a^{2}}{M_{\star}^{2 / 3}} \int_{0}^{t} \Sigma_{\mathrm{s}} d t\right]^{3}$.

This paper has been typeset from a $\mathrm{T}_{\mathrm{E}} \mathrm{X} / \mathrm{L} \mathrm{T} \mathrm{E} \mathrm{X}$ file prepared by the author. 